\title{
An Integrated Home-Based Self- Management System to support the Wellbeing of Older Adults
}

\author{
Julie Doyle ${ }^{1}$, Andrea Kealy ${ }_{3}^{1}$, John Loane ${ }^{1}$, Lorcan Walsh ${ }^{1}$, Brian O’Mullane ${ }^{1}$, Carl Flynn ${ }^{1}$, Rodd Bond ${ }^{1,2}$, Andrew \\ Macfarlane ${ }^{1}$, Brennon Bortz ${ }^{3}$ and R. Benjamin Knapp ${ }^{3}$ \\ ${ }^{1}$ CASALA, Dundalk Institute of Technology, Dundalk, Co. Louth, Ireland \\ ${ }^{2}$ Netwell Centre, Dundalk Institute of Technology, Dundalk, Co. Louth, Ireland \\ ${ }^{3}$ Institute for Creativity, Arts and Technology, Virginia Tech, Blacksburg, Virginia 24060, USA
}

\begin{abstract}
With an ageing population and the constant need towards improving the quality of life for older people in our society, there comes an urgent challenge to support people where they live in an environment that adapts to their needs as they age. While much research on ubiquitous sensor systems and telehealth devices focuses on this need, many of these solutions operate at less than full capacity, and with little scope at present to assess everyday aspects of wellbeing. They focus on detecting sudden critical physiological and behavioural changes and offer few mechanisms to support preventative actions. The challenge of predicting changes and prompting positive preventative intervention measures, aiding the avoidance of severe physical or mental harm, has not adequately been addressed. This paper discusses our experiences of designing, deploying and testing an integrated home-based ambient assisted living ( $A A L$ ) system for older adults, consisting of ambient monitoring, behaviour recognition and feedback to support self-management of wellness, in addition to providing feedback on home security and home energy. It offers a complete system overview of an AAL solution in smart environments and discusses our lessons learned with the goal of assisting other researchers in the field in designing and deploying similar environments.
\end{abstract}

Keywords: Aging in place, smart homes, independent living, older adults, activity recognition, behaviour change, telecare 


\section{Introduction}

Countries globally have been experiencing an unprecedented increase in the number of older adults. From 2006 to 2026, within the 65 and over age group, the dependency ratio is expected to increase from $16.4 \%$ to $25.1 \%$ for Ireland, and increase from $25.2 \%$ to $36.6 \%$ for the EU 25 [15]. In the US, the dependency ratio was $22 \%$ in 2010 and is estimated to climb to $35 \%$ in 2030 [65]. The health statistics released by the Irish Department of Health indicate that average life expectancy of the Irish population has grown by 10 years over the past 50 years [27]. In the US, average life expectancy has grown from 69.9 years for those born in 1959-1961 to 77.9 years for those born in 2007 [10]. Thus, supporting older adults where they live and in an environment that will adapt to their needs as they age, is a pressing need and represents a critical challenge.

As a result there has been an elevated interest in understanding the factors that may support the maintenance of independent living and quality of life of older adults. Smart homes that support ambient assisted living (AAL) have a large role to play in this - particularly in moving from a reactive to a more preventative model of healthcare. Smart homes that support longitudinal monitoring and behaviour recognition enable a better understanding of the causes and the relative contributions and interactions between the different factors that contribute to illness. This is a pre-requisite for early detection, prevention and management and ultimately enables more individually tailored interventions that can be delivered in a timely fashion, resulting in a more individual patient-oriented treatment.

\subsection{The Need for Longitudinal, 'Orange'-Flag Monitoring}

Interventional therapies exist to act on particular signals of ill health. For example, raised blood pressure recordings prompt the ensuing prescription of blood pressure lowering drugs. Blood pressure monitoring then follows within a looped process of target setting and evaluation of variance above or below the set standard. Interventional treatment, when operating in this way, translates easily to the telemedicine context: at one end, discrete data is gathered or sensed by readily available devices, processed via a simple decision tree, and outputted to an action stream linked to clinician or pharmacist opinion. Such systems, whether they apply to blood pressure management, falls detection, or blood sugar assessment, have intrinsic value, yet they do not provide a holistic overview of the person's general wellbeing, including emotional state, fatigue, somnolence, and cognitive performance. Such determinants represent key predictors of quality of life and related mental health in older person cohorts, having a similar magnitude of effect as diseaserelated disability [22] - yet it is the latter component which has dominated interventional telemedicine systems of care as they easily permit the distinct delivery of a specific intervention in response to a significant, macro-event.

By way of example, an alarm-activated fall occurrence precipitates a nurse visit, which in turn precipitates an emergency admission. Thus a red flag process (an event that requires immediate action) is detected and enacted. An acutely stressful, potentially life-threatening event, with a financial consequence for the healthcare system has occurred. However, the personal fear and anxieties that may have preceded, and indeed follow, the red flag incident are ignored. Such fears and anxieties constitute examples of so-called "orange flag" healthcare data. We define orange alerts as those which don't require immediate action, but rather may be indicators that something is changing and thus might require intervention. For example, if a person leaves the home less regularly than they normally do, this may indicate an orange alert.

There is obvious purpose in detecting such emotional responses: for the individual concerned living in fear of a health event such as a fall, it may create a sufficiently strong motivation so as to force the person to give up their home, and seek a supervised environment, possibly within an institutional care setting - a series of life changing events has thus occurred. Furthermore, there is also obvious purpose in intervening based on an orange flag alert in so far as medical morbidity, social disruption, and healthcare system expenditure is avoided, and crucially for the individual, an improved state of wellbeing is offered. This paradigm allows an AAL environment to remain focused on key metrics of ill health and disease status (so called pathological mode) on one level, but to additionally operate within a novel tier in which psychological, behavioural and other aspects of daily functioning (the psychosocial mode) are recorded, analysed and managed. 


\subsection{A Closed-Loop Integrated System}

The overall goal of our research is to develop a home-based, closed-loop system designed to apply innovative behavior pattern recognition algorithms utilising multi-modal ambient sensor data acquisition to wellbeing state change, and to deliver informational feedback and interventions to further support personal wellness management. More specifically, our aims are to (1) Develop a framework to model wellbeing change in the home and to implement and test novel behaviour-pattern recognition algorithms to predict state change based on multi-modal ambient sensor data acquisition and analysis; (2) Assess the performance and predictive power of the ICT system in real-world contexts through comparative analysis with 'ground truth' state change data gathered through daily self-reported wellness indicators implemented on interactive devices and quarterly wellbeing assessments gathered through validated wellness instruments in the home. (3) Deliver informational, educational and interventional feedback to the person themselves that will support them in managing their wellbeing and that will encourage behaviour change where necessary to promote positive wellbeing. (4) Investigate the technology acceptance and timeliness and effectiveness of the feedback mechanism design within the context of wellness self-management in the home. We believe that an integrated system that supports the provision of feedback to the person is essential to support the older person in selfmanagement of their health and to ultimately improve their quality of life as they age.

Our research takes place at Great Northern Haven (GNH) - a development of 16 smart homes located in Dundalk, Ireland, where older people have been living since June 2010. The contribution of this article is to highlight our work in GNH and to discuss lessons learned in designing and developing our integrated, home-based self-management system to support enhanced wellbeing of the residents who live there. To date, following 3 years of research at GNH, we have completed aims 1-3 above, and thus the article largely focuses on these. We are currently investigating the effectiveness of feedback we provide to residents on their wellbeing. However, we have already collected much information and feedback from the residents themselves concerning how effective they perceive the feedback to be, as well as how they find living with and using the technology in their homes.
The remainder of this article is structured as follows. Section 2 discusses related work in the area of smart home research. Section 3 outlines our work at the GNH smart homes, including our sensor network, sensor validation, and how we feed this information back to residents living there, essentially closing the loop in AAL systems. We also provide feedback from GNH residents, gathered through multiple focus groups, interviews and technology evaluations. Two case studies relating to supporting healthy sleep and positive emotional wellbeing of GNH residents are presented in Section 4. These case studies highlight how we monitor changes in sleep and emotional wellbeing and how we deliver this information back to the residents. A discussion of lessons learned from our research is provided in Section 5, while Section 6 concludes and outlines some areas of future work.

\section{Related Work}

This section presents an overview of smart home initiatives, mostly aimed at enabling ageing-in-place, which have recorded data from residents living in their living labs over multiple weeks. Such studies range from a single highly sensed residence to a series of assisted living apartments kitted out with various unobtrusive activity detection technologies. Often these environments record the daily patterns and behaviours of its residents through a number of sensors, and intelligent algorithms have been created which automatically identify these behaviours. Significant research currently being undertaken is focussing on the extraction of activities of daily living (ADL) from such data, and cases where unhealthy behaviours are found are used to guide interventions.

\subsection{University of Missouri - TigerPlace}

TigerPlace is a series of 32 private apartments designed to facilitate ageing-in-place [62]. It consists of a network of wired and wireless sensors that monitor proximity, motion, listen for audio alerts made by the residents, measure vital signs using multiple sensors (including bed sensors), track resident's gait using the Microsoft Kinect platform, and detect cooking patterns using temperature sensors. A goal of this project is to develop technologies that detect functional decline and/or alert care providers should an adverse event occur. 
Results from the analysis of this smart home data are mainly used to inform clinicians and care providers of changes in overall health status.

\subsection{University of Texas at Arlington - MavHome}

The Managing an Adaptive Versatile Home (MavHome) project focuses on creating a smart home that maximises comfort and minimises cost through predicting the behavioural patterns of the inhabitant, the periodic monitoring of vital signs, water and device usage, use of food items, exercise regimen and medication intake [3], [16], [28]. Proposed applications include monitoring deviation in lifestyle trends and for detecting anomalies which may indicate a sub-optimal health status [28]. Such information would be used to inform clinicians. Another proposed application is a daily reminder assistance system for use by the resident [28].

\subsection{University of Florida - GatorTech Smart House}

The GatorTech Smart House is a smart home developed by the University of Florida that combines a large number of sensors including under-floor pressure sensors, RF localisation systems (to be worn by occupants), a smart mailbox, automatic front door unlocking (for residents), automatic blinds, a smart bed (for extracting bed-restlessness and sleeping patterns), an interactive mirror for communicating with residents (particularly for medication prompting), and smart services for food preparation [24]. The purpose of this environment is to support independent living and the remote monitoring of patients, much of which is based around activity recognition and event detection and is used to inform clinicians of a resident's health status.

\subsection{University of Colorado - Adaptive House}

The Adaptive House at the University of Colorado has been developed to dynamically predict future behavioural patterns of the use of lights, heating and temperature by its residents [42]. Machine learning (neural networks) techniques are used to infer patterns, from the ambiently monitored environment, with the goal of reducing energy costs with minimal user interaction.

\subsection{Georgia Institute of Technology - Aware Home}

The Aware Home at Georgia Institute of Technology is a three story smart environment which investigates the design, development and evaluation of future domestic technologies with the overall aim of enhancing quality of life and lengthening lives [32], [33]. Models of user habits and behaviours are generated using sophisticated mathematical techniques and behavioural patterns extracted using location information of the resident (collected via a smart floor), cameras and microphones [32]. This environment allows for the investigation of the longitudinal changes associated with ageing, interventions, and for the supports required to allow ageing in place.

\subsection{Massachusetts Institute of Technology - House $n$ PlaceLab}

The MIT House_n/PlaceLab project investigated using a highly sensed smart apartment ADL detection, user prompting (for labelling of activities) and machine learning based inferencing engines [26], [37], [64]. Data were collected from contact sensors on doors, windows and drawers, electrical flow sensors, light sensors, pressure sensors, gas sensors (for heating and cooking), water sensors, 277 motion sensors placed on objects, RFID tagging, two 3-axis on-body accelerometers, and infra-red motion detectors. Video and audio data were collected from all rooms excluding the bathroom. 98 different activities were identified using multiple data sets including a couple living in the apartment continuously for a duration lasting ten weeks. Much of the research performed during this project focussed on the development of automated algorithms for ADL detection. Subsequent research focussed on addressing the challenge of providing 'just-in-time' information and prompts to the user in order to guide healthy behaviours [26].

\subsection{Washington State University - CASAS}

The CASAS Smart Home Project at Washington State University is a three-bedroom apartment testbed whose priority is to improve the comfort, safety and/or productivity of the its resident(s) [14]. The smart apartment data are used to generate models of user behaviour and recognise activities [14], [11], [54]. These data are recorded from multiple sources including motion sensors placed 
approximately 1 metre apart throughout the entire house combined with temperature readings, water and stove usage, phone usage, and contact switch sensors, monitoring interactions with the telephone directory, the cooking pot, the medicine container, and important ingredients. An important aim of the CASAS Smart Home is to perform functional health assessments longitudinally using ambient sensors, to provide user prompting for ADLs as required, to assess the resident-acceptance of the technologies, and to evaluate the benefit to caregivers.

\subsection{Oregon Health and Science University - ORCATECH}

The ORCATECH Living Lab consists of a group of community dwelling older adults, that have had unobtrusive monitoring technologies installed in their homes since 2006, who provide a test-bed for evaluating behavioural monitoring technology [31]. Technologies installed into the homes include motion sensors, contact sensors (placed on doors and cabinets), bed movement sensors, motion-based gait speed detector, phone sensors and medication usage detectors. The 30 participating seniors are evaluated by clinical and neuropsychological tests. In addition, the characteristics of their home environment (type of home, home construction materials, number of rooms, etc.), and of their socialization habits (regular outings and visitors) are documented. Every subject also completes a short weekly online questionnaire.

Subsequently, these technologies were rolled out into the homes of 265 older adults in the Intelligent Systems for Assessing Aging Changes (ISAAC) study, and concomitant health and functional questionnaires, physical examinations, and neuropsychiatric testing [31]. Initial results demonstrate the feasibility of the large-scale community deployment of in-home activity assessment technology.

The information extracted from the technologies deployed into the homes of these older adults may be used to both inform clinicians of a change in health status, and to connect older adults with a remote health coach and remote family members [29].

\subsection{SI4Life}

The SI4Life Consortium provides a living lab in Milano, Italy, known as the "DAT project" [61]. It consists of 10 smart homes designed and developed to educate people with disabilities as well as their family, how to live independently using technological innovations.

The SI4Life living lab provides homes with state of the art solutions in home automation and AAL, to subjects with disabilities (cognitive, neuromuscular, neurological, orthopaedic etc.) in order to (A) Test technologies and to identify which best support the specific condition of the subjects, and (B) Educate the subject and their family to use these technologies, with the paradigm of learning by doing, in order to increase the level of independence and the quality of life of the subjects.

The apartments are around 130 sq.m. each with seven different rooms plus a room for data equipment, acquiring an elaborate array of ambient and biological signals. In addition to the existing 10 homes in the Si4Life living labs, it is planned to expand to further homes in Liguria, Italy.

\subsection{Other Research Initiatives}

In addition to research concerning specific purpose-built smart homes, there are a number of other research initiatives using ambient sensing to detect behaviour and changes to behaviour that might be indicative of changes in health status. Work at the University of Amsterdam is examining how best to detect functional health status of older adults using ambient sensing [56] as well as how to visualise such information to ensure it is relevant to caregivers [55]. Steen et al. [63] use ambient sensing and models of human behaviour to detect anomalies, or abnormal behaviour, in home environments. A field trial run in two older adults' homes over an 8 month period determined that the models used were capable of describing human behaviour within real world environments, but were not suitable for all rooms in the home, suggesting a varying nature of activities from room to room. Other work has focused more narrowly on monitoring and detection of changes for specific health conditions. COMMODITY12 uses a combination of ambient and wearable sensing and portable devices to monitor physiological data and health-related context information, including physical activitiy and and vital body signs in diabetes patients [30]. Analysed data is presented back to the patient as well as to their caregivers. 


\subsection{Summary}

Many research groups are investigating the development of AAL-type applications using data collected from artificial intelligent lab-based environments (the 'home-in-lab'). While this provides initial data for analysis and facilitates focus groups, the data collected is not representative of the real world. Initiatives, such as those listed above, enable researchers to investigate the real-world living patterns from individuals over extended periods. The duration of time required to capture the overall variation in such behaviours and tasks is unknown. Most of the studies collect data when people are staying temporarily in their living lab with the exception of ORCATECH and TigerPlace who are collecting real-world data from people living in their homes. The remainder of this paper describes the living lab at CASALA, a series of 16 apartments, where residents have been living since June 2010. One of the unique features of $\mathrm{GNH}$ is that information learned from ambient monitoring is returned to the resident themselves (rather than a carer or clincian) - empowering them to self-manage their wellness and their home's security and energy usage.

\section{The Great Northern Haven Smart Homes}

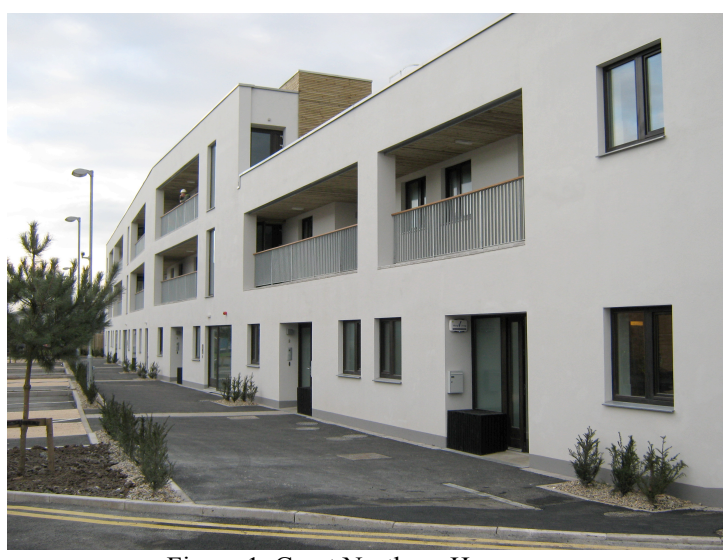

Figure 1. Great Northern Haven

Great Northern Haven (GNH) is a demonstration housing project consisting of 16 purpose-built smart homes in the north-east of Ireland (Figure 1), each equipped with a combination of sensor and interactive technology to support AAL for older people. Fifteen of the apartments are occupied by older adults, while one is retained as a unit for demonstration and testing purposes. This $16^{\text {th }}$ apartment is also used as a transitional unit to support older adults moving from high dependency care back to their own homes. Ambient sensor technology supports monitoring of patterns of behaviour over extended periods of time, and more importantly deviations in normal patterns of behaviour that might indicate the onset of illness (orange alerts).

To date, residents have been living in GNH for three years (since June 2010). Thus we have gathered large amounts of data from sensors in the 16 apartments, and validation and analysis of the data has taken place. Models are being built to detect patterns in activities of daily living and health. GNH is a unique development in that it is not a testbed for research. These are real peoples' homes and as such, the data we are collecting is extremely rich. We work very closely with GNH residents in determining their needs. They are not research volunteers, as is the case with most research projects (the residents moved into GNH based on health or housing needs), and this provides us with a unique setup. Furthermore, the residents represent a diverse cohort, ranging in age from early 60 s to late 80 s, representing varying levels of computer use and interest, and each with varying health conditions. We are currently beginning to feed information we are ambiently gathering back to residents to support them in managing their wellbeing and homes - essentially closing the feedback loop in AAL. This is achieved through interactive devices such as the iPad, hosting a suite of applications ranging from health and wellness, to home security and energy. An overview diagram of our system is shown in Figure 2. Environmental sensing, behaviour recognition algorithms and ground truth data are combined to determine an intervention approach. Feedback and validated interventions are then deployed to the resident. This closed loop mechanism of sensing and feedback is currently in place at GNH. Our ongoing and future research is examining the effects such feedback has on wellbeing and behaviour change.

\subsection{GNH Sensors and Actuators}


There are a total of 2240 sensors and actuators throughout the GNH development, with approximately 100 sensors embedded within each home and further sensors in public places, such as stairwells and outside the compex. The sensors include passive infra-red (PIR) sensors to detect motion; contact sensors on all windows; contact sensors on all exterior doors; contact sensors on three interior doors; sensors on all light switches; temperature sensors for each room; brightness sensors at three points in the homes; weather data including outside brightness, temperature, wind speed and rain alarm; sensors to detect power consumption and heating usage. In addition each change. PIRs, light switch sensors and temperature sensors report only on change. The type of reporting has consequences which will be discussed in the data validation section below.

While the number of sensors is very high, part of our research involves examining which subset of sensors are the best indicators of changing conditions, for example what are the 5 optimum sensors that might be retrofitted to existing homes to support AAL.

Home automation also plays a role in Great Northern Haven. For example, the height of the sink and of the cooking hobs can be changed to suit the resident's needs. Additionally, many windows and

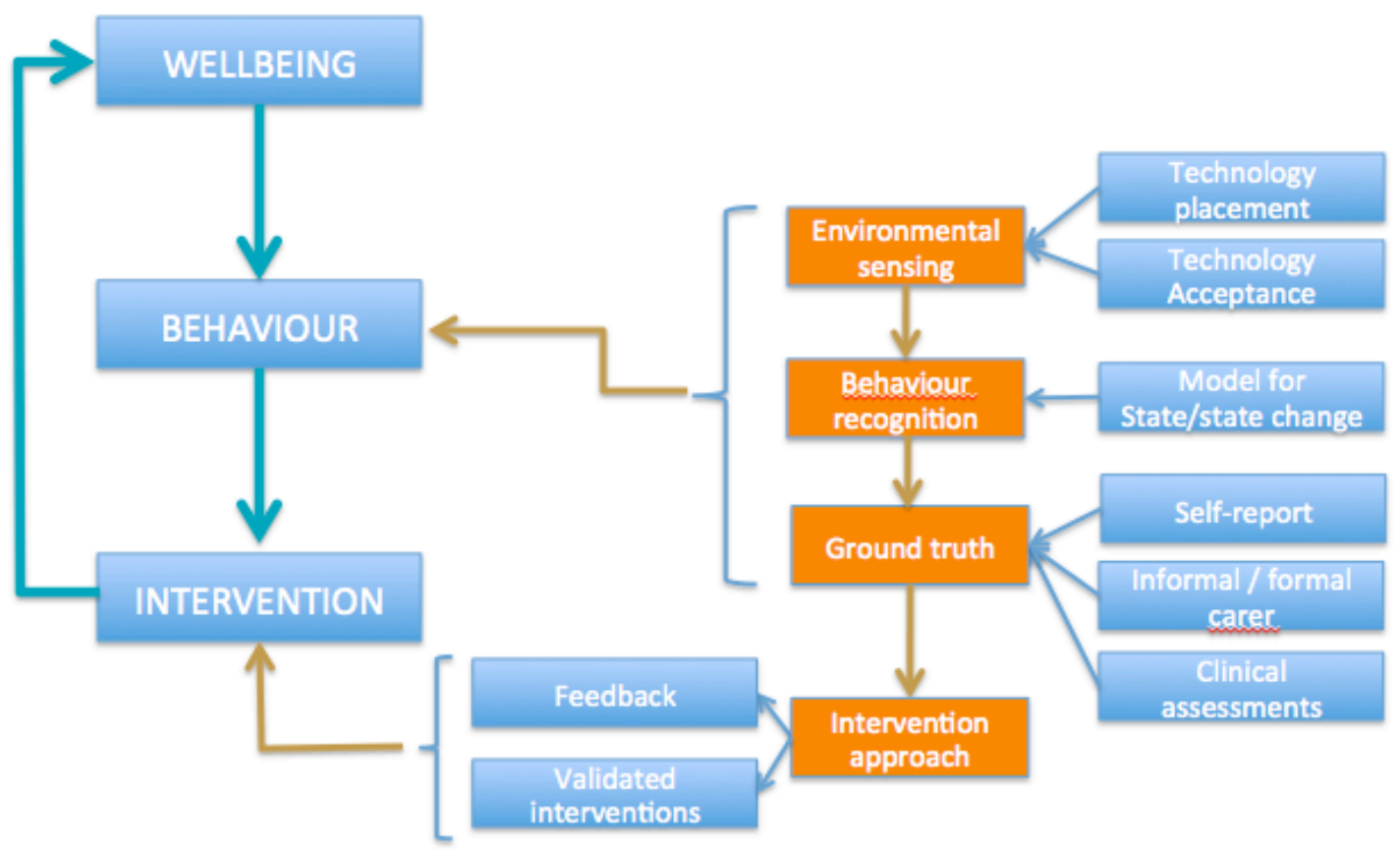

Figure 2. System Overview

home has a number of alarm cords and buttons, a home security system and a telecare device that links with a monitoring service.

Sensors report (A) periodically, (B) periodically and on a change or (C) solely on a change. Some sensors, such as interior light levels, can be configured to report once light levels change by a certain threshold or periodically. It is also possible to set the resolution of the periodic reporting and sensors are set to the highest resolution available. In GNH the electricity sensors report every 12 seconds, outside light levels report every 10 minutes and internal light levels report every 5 minutes. The contact sensors on doors report periodically every 15 minutes as well as on doors can be opened and closed automatically, and an intercom system allows the resident to view, and talk to, a caller before remotely unlocking the front door. A 'TV style' remote control with a limited number of buttons may be customised to allow residents to use any of these services (such as opening a window or door, switching on or off lights, or opening window blinds). 


\subsection{Sensor Network Architecture}

GNH uses a wired Konnex $(\mathrm{KNX})^{1}$ network to gather all of the sensor data. The data is gathered using KNX sensors and aggregated and logged by NETxAutomation OPC server software. The data is written to a local Microsoft SQL Server 2008 r2 Express edition database as well as being written to $\log$ files once a day. Each of these services runs on a dedicated server in GNH. We replicate all of the data from the local database in GNH to a Mysql server in our research centre with a script that runs every fifteen seconds and connects via a Virtual Private Network (VPN). As the Express edition of SQL Server has a maximum database size of $4 \mathrm{~GB}$, it is important to clean out the database regularly once the data has been replicated to our Mysql server. At 5am each day a script is run which cleans out records from the SQL Server database that are more than 120 days old and which have already been copied to the server.

The replication script copies up to 400,000 records a day into a daily table on the server. As the replication script runs every fifteen seconds, data on the Mysql server is close to real time. An important issue with dealing with this many records is the size of the data on disk. A daily table with 400,000 records takes up almost 54MB of disk space. It is important to keep the tables as small as possible so that data can be quickly inserted. At 3 am each day data is aggregated from the daily table into a monthly table. The raw data from the daily table is processed before being added to the monthly table. Sensor id's are converted from varchar format to smallint so that they take up less space on the disk. For the same reason, two fields containing address information for the sensors are dropped from the daily table and replaced by an apartment id field. With this processing done, a typical monthly table of records is $500 \mathrm{MB}$. This is small enough to be loaded into memory in one block and hence database queries run fast. Where previously, the input database table had grown very large, database queries ground to a halt due to disc swapping as the table was too big to fit in memory.

A script runs every 30 minutes to check that data is being recorded on the SQL Server database in GNH. If the last entry recorded in that database is more than one hour behind the current time, an email is sent to the team to ask them to check that all systems are

\footnotetext{
${ }^{1}$ http://www.knx.org/knx-en/index.php
}

operating correctly. If the internet connection to GNH is interrupted, the replication script automatically copies all unreplicated data once the connection is restored.

Once we have the data copied to the server and in tables that are partitioned to be small enough so that queries run fast we process the data. Data processing is achieved by scripts run as cronjobs on the server. Each of these scripts writes data to summary tables in the database. This data is then served to end user applications through web services which output data as JSON.

\subsubsection{System Data Loss}

System data loss has resulted from a number of factors, including using the express edition of SQL Server in GNH. This has a maximum database size of 4GB. When this is exceeded the database write fails. The solution is to clean out records more than 120 days old from this database once they have been replicated to the server.

The backup for the previous problem is to ensure that $\log$ files are also written to the server in GNH. One issue with this is that the default KNX setup wrote to a single file which once it reached a certain size started to overwrite the oldest records. The solution was to reconfigure the system to write a new $\log$ file once a day at $3 \mathrm{am}$.

The boxes in GNH are Windows machines, which by default are set to automatically restart the machine to install updates. This causes data loss and the solution is to disable this feature of Windows.

Individual sensors may fail. Of the 16 homes in GNH only one PIR has failed in a 3 year period, reporting nothing. Other problems include corrupt sensor readings. This will be discussed in the next section on data validation.

Finally, over verbose logging may result in data loss. The replication script runs every 15 seconds and a number of processing scripts run once a minute. When these scripts fail to run they can produce a massive amount data in the log files which quickly uses up all available memory on the server and causes it to crash. The solution to this is to limit the amount of logging in the scripts and rotate the log files once they reach a certain size.

\subsection{Sensor Data Validation}

Significant validation has been carried out on all sensor data to ensure the integrity of the data is maintained. This section outlines some of the 
validation checks performed. The complexity of the validation is dictated by how the sensor reports. Periodic sensors are the easiest to validate as they should report a constant number of records per day.

The first of these quality measures relates to data loss measured by periodic sensors, specifically in verifying that we are not missing data from any one day. Each apartment has one electricity sensor which should fire every 12 seconds. By verifying that each individual electricity sensor has approximately 7200 records for each day, this provides strong validation as to whether data has been lost or not. If there are significantly less electricity records it could mean that the system crashed and will result in other sensors suffering from similar data loss. Brightness sensors, which are also periodic and fire every 5 minutes for internal sensors and 10 minutes for external sensors, provide similar validation. As these sensors are all periodic this validation is strightforward - we expect to see a constant graph when plotting totals from periodic sensors.

The second type of sensor that we validate are those that fire periodically and on change. In our set up this includes all windows, internal doors and external doors. These contact sensors fire every 15 minutes or on-change. Hence each of these sensors should fire at least 96 times per day.

The final type of sensor is that which fires only on change. In GNH these include PIRs, temperature sensors, contact sensors on light switches and a wind speed sensor. PIR sensors may be verified against each other. For instance no two sensors should pick up the same movement. Secondly, transitions between rooms should be detected. If, for example, movement ceases in the living room we would expect the PIR in the hallway to detect movement prior to the PIR in the bedroom. If two of the PIRs fire greater than ten times and the other does not fire at all then we may have a problem. The threshold of ten is to allow for cases where residents are away from their home but someone calls to briefly check on it. The PIR sensors used in all apartments in GNH output values of 1 or 0 . Each PIR sensor should fire with a value of 1 when movement is detected, changing to a zero value after motion has stopped. The PIR sensors issue an end of movement output value of 0 exactly 10 seconds after the last detected movement. This overshoot time has been validated experimentally. The temperature, light switch and windspeed sensors can be validated by looking at a sliding window of the last thirty days to assess whether the number of firings falls within typical ranges.
Aside from quality checking, visibility of PIR sensors has also been assessed through an experimental validation. The validation was carried out in each room containing a PIR, as well as adjacent rooms in the PIR's line of sight. These areas included the open plan living room and kitchen; the hallway, as well as the entrances to the toilet and guest bedroom; and finally the main bedroom and en suite. Rows of points were marked with tape every 14 inches parallel to the wall holding the PIR sensor, see Figure 3.

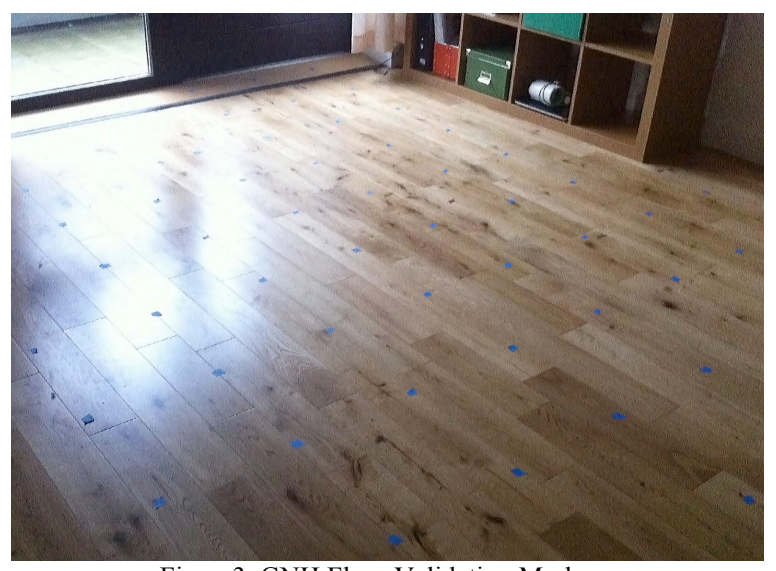

Figure3. GNH Floor Validation Markers

Continuous motion, from the waist up, was undertaken on each point for the overshoot time plus an additional 5 seconds to ensure that the movement was measured on the point, rather than to the point. This method was carried out on all points parallel to the PIR bearing wall, following the red lines in Figure 4, and also diagonally, along the blue lines.

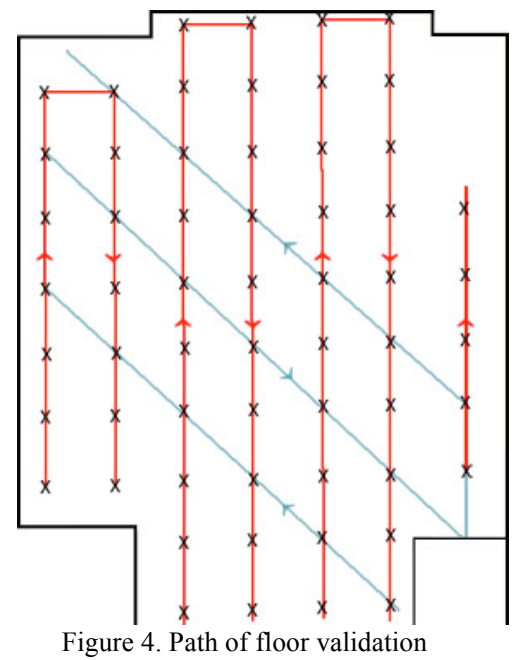

Figure 4. Path of floor validation 
The blue areas of Figure 5 show areas that remain undetected by the PIRs. These include the row closest to the wall bearing the PIR; the corner by the rear door and most of the kitchen area. Areas which detect movement towards them but don't detect subtle movement within them (the PIR fired on moving to the spot but then shut off after a few seconds) are coloured light green. Areas completely visible to the PIR sensor, are shown in darker green.

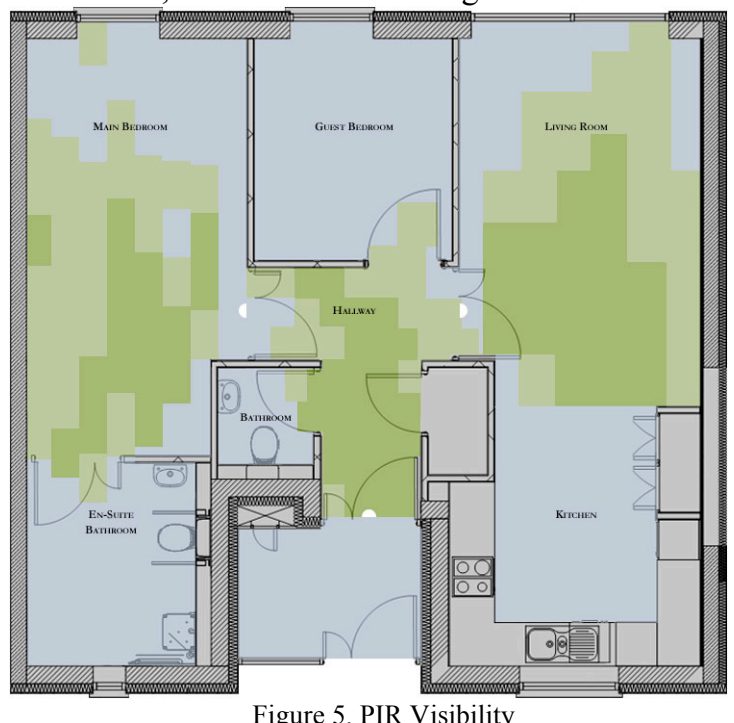

Figure 5. PIR Visibility

A number of additional PIR sensors have been added to our test/demonstration apartment at GNH to achieve a higher level of visibility. Figure 6 shows the increased visibility these sensors identify which facilitate validation of the location mapping algorithm discussed in section 3.4.3. We are planning to introduce two of these additional sensors into the other apartments of $\mathrm{GNH}$ - one in the en-suite bathroom and one in the kitchen.

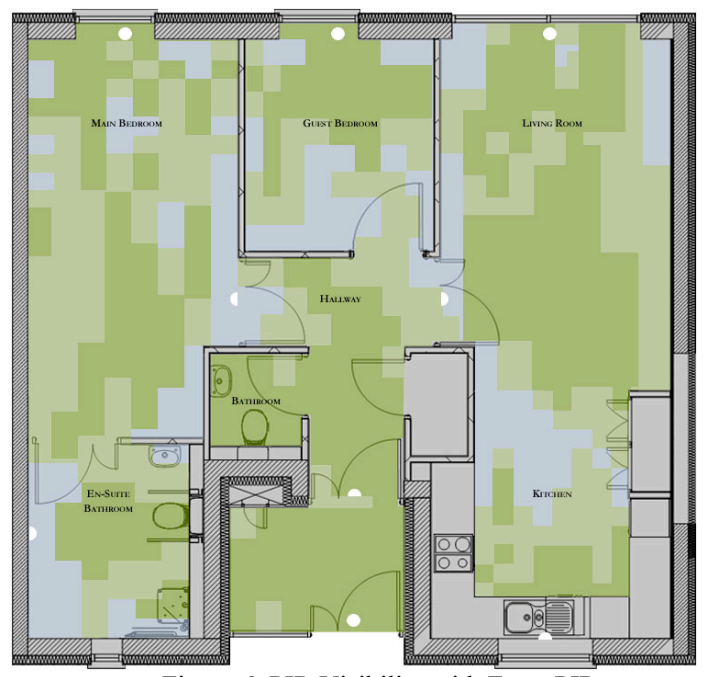

Figure 6. PIR Visibility with Extra PIRs

\subsection{Models/Feature Extraction}

The nature and volume of the sensor data is complex and large. Various features are extracted to facilitate further analysis. We currently have scripts running once a day which calculate the total energy usage for the previous day; and total movement duration in each apartment per day, hour and minute. We also have scripts that run every minute to calculate hourly electricity usage; check whether there are any windows or external doors open or whether any high energy appliances have been left on for more than 10 minutes; calculate current weather statistics; and rotate $\log$ files. Further algorithms have been developed to extract the time outside the home, location mapping and sleep metrics.

\subsubsection{Residents' Movement Levels}

An algorithm has been developed that measures the residents' movement per minute, hour and day. This is calculated using the three PIRs installed in each apartment. In calculating the movement time we have subtracted the 10 second overshoot time from each PIR firing interval to ensure the movement duration covers only real movement.

Variations in resident's movement levels often reflect changes in a person's behaviour. For instance, previous research [48] has highlighted instances where an increase in resident's movement at night time was detected directly prior to hospitalisation. Currently this algorithm is only valid for single occupancy in the home. Peaks in movement during the day may be indicative of a visitor being present, but more work is required to distinguish between 
higher than normal activity for a single occupant versus normal activities with visitors. As part of selfreporting, we have recently begun to ask residents to report when they have visitors. This is being run for a short trial period after which we will compare sensor data with visitor reports.

Decreases in movement while a person is in the home may indicate a decline in health or, alternatively, a change in the way a person uses their home. Feeding this data back to the resident can be useful in providing context to the changes which occur.

\subsubsection{Time Outside the Home}

Time outside the home is important in considering the sociality of an individual. It may be calculated by subtracting the time a person closes an external door from the time they open an external door, providing no PIR has fired with a value of 1 during this period. While time outside the home isn't directly fed back to the resident, it is used in determining the resident's emotional wellbeing, further discussed in section 4.2.

\subsubsection{Location Mapping}

A location mapping algorithm has been developed, employing a subset of sensors installed in GNH. Figure 5 illustrates the standard placement and visibility of PIR sensors, located in the hallway, living room and main bedroom. The location mapping decision model, as depicted in Figure 7, uses PIRs to establish an initial position for an individual. As the PIRs installed in GNH have been validated to detect transitions between rooms it is possible to deduce an accurate initial position from which to check whether the resident remained in the room in which the most recently fired PIR is located, or whether movement continued to an adjacent room in which movement is undetected by the PIR.

The location mapping is used in deriving metrics, such as those relating to sleep and time outside the home and, as such, isn't fed back directly to the resident.

\subsubsection{Sleep Metrics}

Various sleep metrics have been derived by considering elongated periods within the bedroom, as depicted in the location mapping algorithm. Metrics include estimated bed time and estimated rise time, sleep disturbances and time in bed. Bedroom and out of bedroom events, calculated by the location mapping algorithm, are examined in determining the estimated sleep window, i.e. the period within which the bed time and rise time are located. If an out of bedroom event occurs and the duration of time spent in the bedroom directly following this disturbance is

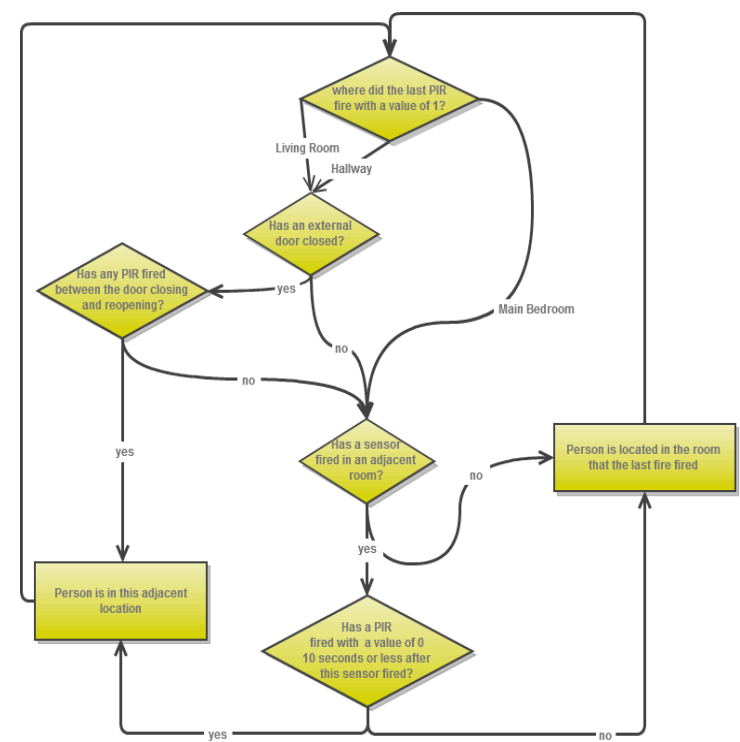

Figure 7. Location mapping model

greater than a certain threshold it is deemed to be within this window, otherwise the end of the previous period in the bedroom is deemed to be the end of this sleep window. This is repeated in reverse to detect the start of the estimated sleep window.

Once an estimated sleep window is determined for a given night the movement levels at the beginning of this window are used to estimate the bed time and rise time. If movement is below a subject-specific threshold for a period of ten minutes or more, the start of this period is determined to be the subject's bed time. Rise time is estimated by deducing the last period at the end of the sleep window whereby movement is below a subject-specific threshold for a period of ten minutes.

Sleep disturbances are calculated by finding periods of movement above a certain threshold within the bedroom, as well as movement out of the bedroom between bed time and rise time. Time in bed is then calculated as the time between bed time and rise time, removing periods which were calculated to be sleep sleep disturbances.

Once validated, the above metrics will be integrated with resident's self reported sleep data to feed back a sleep score to the user through the iPad application, YourWellness, discussed in Section 3.5.1. 


\subsubsection{Electricity Usage}

Electricity usage is continually logged to a daily table on the server and aggregated nightly to a monthly table, as discussed in section 3.2. Cron jobs are run every minute to calculate their hourly usage, and each night to calculate daily usage, for each apartment. These are fed back to residents through an electricity monitoring app, discussed in more detail in section 3.5.3.

\subsubsection{Weather}

A weather summary is scraped from the national weather website and local weather data gathered at GNH is used to calculate maximum, minimum and average values for temperature, wind, light levels and percentage rain during the day. Current weather statistics are calculated every minute and fed back to the user through a weather monitoring app. A daily summary of weather statistics is also saved in the database, which may be used in future work analysing the affect of weather on individual's time outside the home.

\subsubsection{Ground Truth}

Gathering ground-truth data is essential to help us understand and make sense of the ambient sensor data. It allows us to assess the performance and predictive power of the system through comparative analysis with 'ground truth' state change data gathered through daily self-reported wellness indicators implemented on interactive devices, and quarterly health and wellbeing assessments gathered through validated wellness instruments in the home.

Self-report. Self-reported data is one way in which we gather ground truth, helping us to label ambient sensor data. An iPad application has recently been implemented to support GNH residents in this (discussed further in Section 3.5). Within a selfreport application, individualized questions can be asked of participants to help us understand their repeated patterns of behaviour, in addition to gathering their perceptions on their wellbeing.

Clinically validated questionnaires. The GNH residents answer a battery of questionnaires quarterly to further support validation. Measures include the Center for Epidemiologic Studies Depression Scale (CES-D) [53], the Hospital Anxiety and Depression scale (HADS) [70], the Montreal Cognitive Assessment (MOCA) [44], the 36 item short-form health survey (SF-36) Quality of Life questionnaire [66], the De Jong scale of emotional and social loneliness [18], the Instrumented Activities of Daily Living (IADL) scale [36] and the Pittsburgh Sleep Quality Index (PSQI) [7]. These questionnaires are administered by a researcher in the resident's home.

Ongoing work involves examining correlations between all ground-truth data and the sensor data. This is discussed further in Section 4.

\subsection{Feedback Applications}

A crucial aspect of our system is returning information that we learn about the residents to them - essentially closing the loop in AAL and empowering the resident to manage (1) their wellbeing, (2) their home's security and (3) their energy usage. iPad applications have been developed to support this. Throughout the process of designing these applications, we have worked closely with the residents of GNH - from application concept, through to design and usability evaluation. While this paper mainly focuses on health and wellbeing, home security and home energy applications are also discussed in this section as they provide feedback to the residents of GNH.

\subsubsection{YourWellness}

The YourWellness application is an $\mathrm{iPad}$ application to support the person in self-reporting on aspects of their wellbeing and to provide feedback on such, in terms of showing an overview of an individual's wellbeing, trends of their data over time and information and education on how to maintain or return to a healthy state of being.

In designing this application, it was critical to involve older adults, to understand their attitudes towards wellness, as well as assessing the effectiveness of various types of input and feedback visualisations. We conducted a number of focus groups with older adults as part of the design process, whereby we received feedback on methods for inputting information, preferred visualisations of feedback and the type of content that would be required for such an application [20]. We also ran workshops with clinicians to ensure the application's validity as a wellness tool.

The application currently asks participants to selfreport on their mood, sleep, social interactions and provides a daily trivia question. Blood pressure and weight management are supported through Withings devices that interface with the iPad. More recently, we have added falls history and fear of falling to the 
application. At the highest level, feedback is provided as a quick-glance overview of wellbeing. Based on feedback from participants of our focus groups, we have designed a feedback wheel to support this (Figure 8).

The wheel is divided into segments, based on the parameters of wellness that are being monitored. The interior part of the segment is coloured green if the individual is considered healthy, meaning they don't need to take any action regarding behaviour change for that parameter of wellbeing. If the individual is scoring relatively low in a particular area of wellbeing, the segment is coloured amber - indicating an orange alert and that some action should be undertaken to address this. A red segment means immediate action is required, and the individual will be alerted (blood pressure and weight are monitored by a $3^{\text {rd }}$ party triage service). In collaboration with clinical specialists and taking into account existing guidelines such as the NHS NICE guidelines [46] (including 'Treating Depression in Adults' and 'Mental Wellbeing and Older Adults'), we have determined a scoring algorithm that calculates a wellness score for determining whether a green, orange or red alert should be provided. For example, with blood pressure, a person's baseline or 'normal' blood pressure is calculated over a period of two weeks and then deviations above or below this 'norm' indicate a red or orange alert.

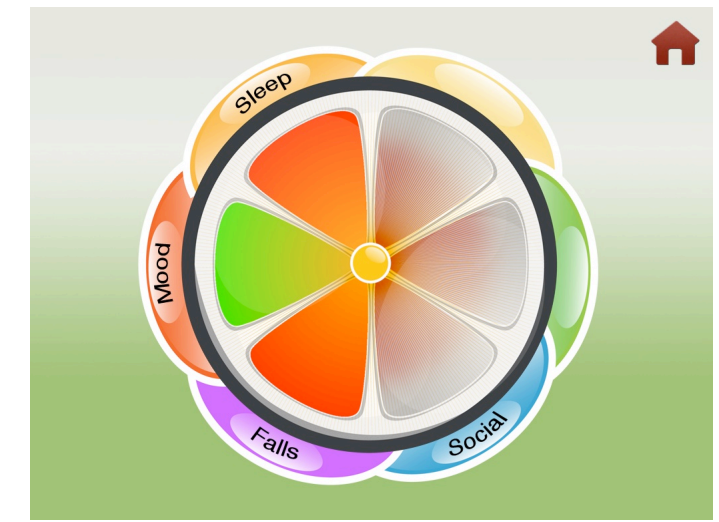

Figure 8 . The feedback wheel with each segment representing a parameter of wellbeing.

An individual can also click a particular segment of the overview feedback wheel to get further information, including their trending/historical data presented as a graph that is viewable as weekly or monthly data (Figure 9). Educational and interventional content is also provided. Initially, this was as a series of messages delivered daily (Figure 10). However, following feedback from residents we now also include multimedia content, in the form of videos and interactive images (e.g. an image of a kitchen with red dots that indicate trip hazards clicking on the red dots provides more information). Such content has been defined for each type of alert in each category of wellbeing, in collaboration with clinicians and by examining existing guidelines. For example, if an individual is scoring in the orange zone for emotional wellbeing, feedback might include encouraging them to go for a regular walk. It might also involve asking additional questions to assess why the person is scoring low. The overall aims of such feedback are to promote awareness of wellness and to help the individual to improve their wellbeing - to move from being in the red/orange zone to the green zone. Indeed, feedback from older adults who have tested this application suggests that simply self-reporting is in itself one level of feedback as it increases awareness.

This application has been deployed for 11 months, since March 2013, in GNH and also in a number of homes in the community, examining attitudes to the app in terms of usability, the intuitiveness of the feedback and its benefit in promoting awareness of one's wellbeing, as well as adherence to selfreporting. Some feedback from an early evaluation of this application deployment are presented in Section 3.6. The application has been updated to reflect our findings and has been deployed to GNH for longitudinal use.

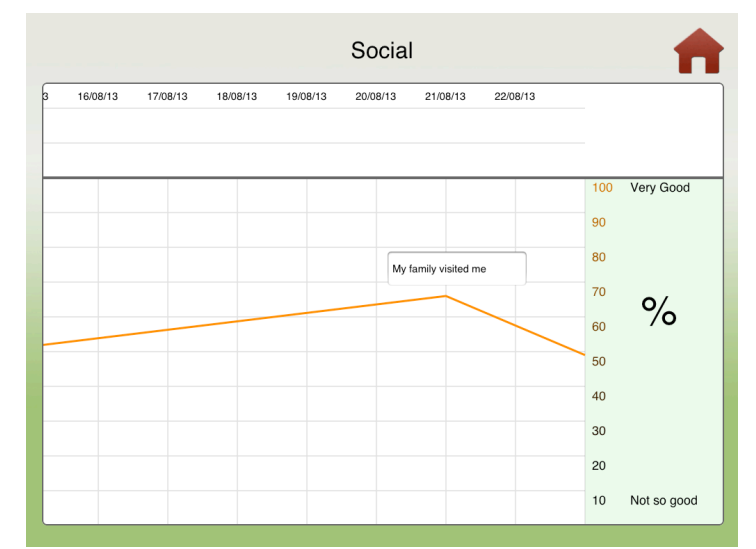

Figure 9. Weekly trend data for a person's self-reported quality of social interaction with annotation inserted to provide context for a particular value. 


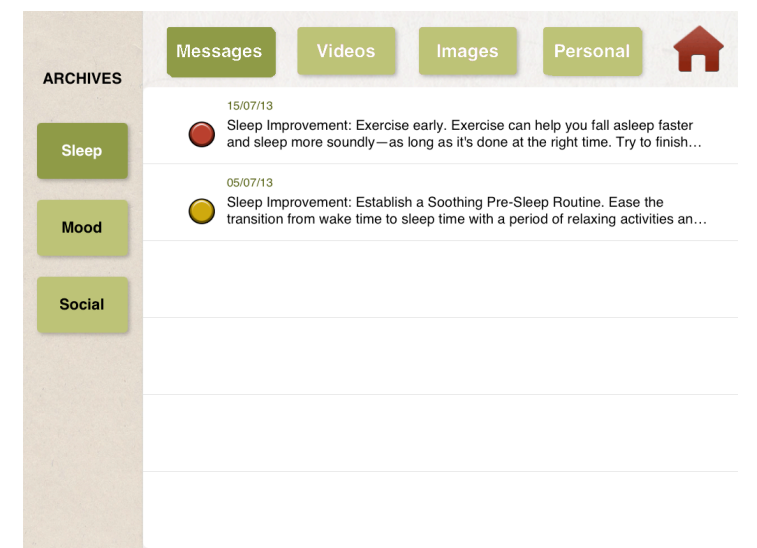

Figure 10. Examples of educational/interventional messages.

\subsubsection{Home Security}

A home security application has been developed that highlights whether a window or door is open/closed, whether the oven/hob (stove) is left on and whether there is a water leak. This application reads information from the door and window contact sensors, water sensor and uses recognition of the electrical signature of the stove to detect open/closed and on/off events. This information is then fed back to the resident on their iPad as shown in Figure 11. The goal of this application is to act as a quickglance overview of the home's security state - that might be glanced at when a person is leaving their home or going to bed at night, for example. As can be seen in Figure 11, if a door or window is currently open, or if the stove is on, the corresponding icon is coloured red. Green indicates a 'safe' state. Clicking on any individual icon brings the user through to more information. For example, clicking on the door icon in Figure 11 will inform the user which door is open. An additional feature that will be added to this application based on resident requests will include feedback on whether common external doors of the apartment complex are open or closed.

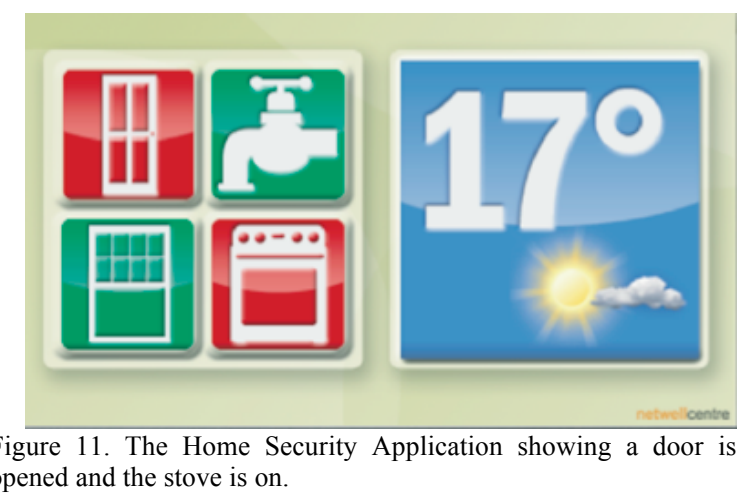

\subsubsection{Home Energy}

An application that GNH residents were particularly interested in was one that computes home energy costs and that provides feedback on the cost of various appliances. The primary reason for this is financial. Energy costs are a concern for many older adults who, particularly in the winter months, worry about using appliances and heating due to their high cost. We want to support the residents in reducing the cost of their home's energy but also to engage them in the process, helping them to understand where savings can be made. Existing energy consumption tools give details on current power usage and / or historical usage, but generally leave it up to the user to interpret what causes the power drain. The application we have developed, shown in Figure 12, provides details on home energy usage and energy costs, helping the residents to identify where they might save money.

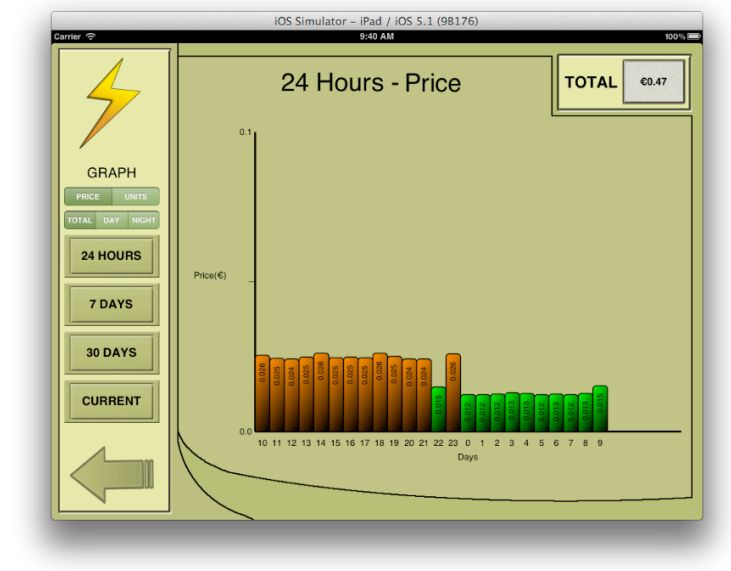

Figure 12. The Home Energy Application showing electricity usage and price for the past 24 hours in the GNH test apartment

As part of our requirements gathering for the energy application, we held a focus group with 8 of the GNH residents, 6 male, 2 female (Figure 13). The focus group asked questions around (1) the residents' current energy usage and their understanding of this; (2) Their interest in saving energy and whether they would like additional feedback on their consumption (other than what they currently receive in their utility bills); (3) What might motivate them to save energy; (4) Gauging their feedback on the intuitiveness of various types of visual feedback of energy data; (5) Whether they would use such an application. Based on feedback from the group, three distinct themes emerged around the areas of promoting awareness, motivation to reduce energy and the application interface. 


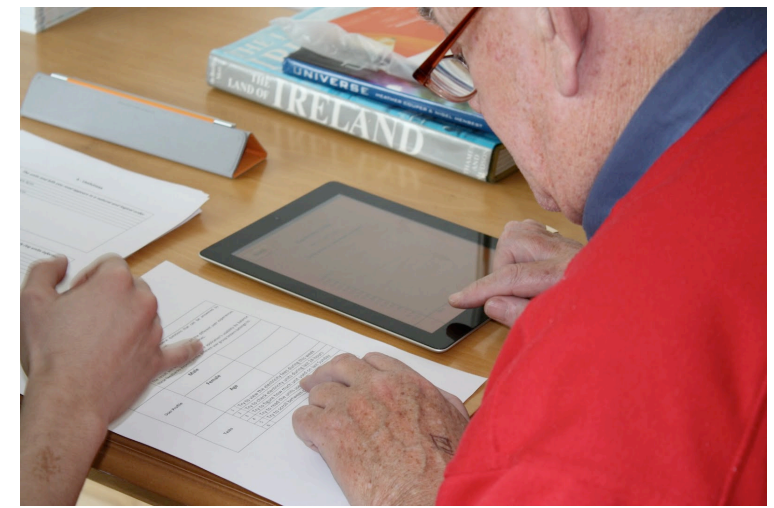

Figure 13. Engagement with older adults

\subsection{Feedback from Residents}

In this section, we report on some feedback we have received from GNH residents resulting from two studies recently carried out: (1) Evaluation of a 4-month deployment of the YourWellness application; (2) Interviews with residents carried out in June 2013 to assess how they feel about living in GNH ' 3 years on'. Detailed results from each of these studies are currently under review elsewhere. However, we provide summaries of our findings here.

\subsubsection{YourWellness Evaluation}

The YourWellness application, as described in Section 3.5.1, has been deployed in homes in GNH since February 2013. Following the first 4 months of deployment, we ran an evaluation to determine peoples' opinion on the usefulness of self-reporting, in addition to the benefits of receiving feedback on wellbeing, in terms of one's own history as well as the educational content. It should be noted that while we are refining this application based on feedback, it is still in use by residents and will continue to be used longitudinally to help us evaluate the effectiveness of such feedback on wellbeing in the long term.

It was felt that the daily questions that were asked as part of the self-reporting aspect of the application were "perceptive and focused" and "they got to the heart of the matter and made you think." It was generally felt that even the act of answering these questions increased awareness of wellbeing. It was felt the graphs were useful to be able to look back over time and "see if your patterns have changed".
Residents reported reflecting on those days or weeks when their scores were lower than normal and trying to remember what else had happened during this time that might have caused this.

The advice messages were overall deemed to be very useful: "I found the messages excellent"; "Really, really good advice, and stuff that I didn't know myself." Residents said they read the messages regularly. However, not all residents followed the advice. Some felt that they already were following them. For example, residents who were particularly active felt they didn't need to do anything additional to what they were already doing. Yet these residents still stated they found the messages useful with one person stating that they "reinforced my confidence in what I was already doing."

We also had a number of suggestions on how to improve the application, to increase motivation to use it and make it more useful. For example, in terms of the self-reporting aspect of the application, one resident described how "initially I enjoyed it very much, but later it became a chore." It was felt having video content as well as text messages would be more engaging. While residents enjoyed the trivia question, they thought that something like a 'thought for the day' or 'joke of the day' would increase motivation to open the application.

The ability to add annotations to graph data was requested, as this would provide context - as to why a person's mood was low on a particular day, for example. People found the questions to be repetitive and would like them to vary more. The categories of wellbeing being self-monitored during this initial deployment were fixed (sleep, mood, social interaction). However, we now have a number of additional categories including falls history, fear of falling, physical activity as well as surveys that are relevant for different conditions, such as COPD, diabetes etc. Therefore, going forward, we can offer residents an application that allows them to selfmonitor those areas of wellbeing that are either of interest or of concern to them personally.

\subsubsection{GNH 3 years on}

Interviews with residents have recently been conducted to determine how they feel about living in GNH 3 years after they initially moved in, and to compare their experience against their expectations, as captured in interviews prior to them moving in. We provide a short summary of our findings here that discuss opinions around the benefits of living in 
smart homes as well as opinions regarding the ambient and interactive technologies.

The main benefit for residents of GNH involves security and safety. This relates both to the security of the home as well as to safety if anything, such as a fall, were to occur. "Well you do like to know that you're safe especially when you go to bed at night.",

"With alarms and sensors you're not frightened.", "Before (moving here) I was afraid of falling in the bath."; "It's peace of mind mostly".

Constant support at hand was seen by many as a benefit: "All this technology and the fact that there's somebody always there (referring to the 24-hour triage amd monitoring service)." "I don't need anybody (carer/family) coming in and I'm doing well."

Residents also feel there are health benefits to living in GNH. "I think I'm going to live for another 10 years. 75 used to be my target. I'll be 75 now in September and my target now is 85." "My health's improved a lot. I'm more outgoing, better than I used to be.". "I'm sorry I wasn't moved in here 20 years ago."

With regard to living with the sensor technology there were some mixed opinions. Overall, residents are comfortable with it. "At first, people thought that there were cameras inside (the sensors) watching them. Now, well I feel at home with it." "That's what they're (the sensors) there for, to make sure we're alright." Two of the residents talked about concerns of "being watched all the time", with one referring to it as a little like Big Brother. As a way of alleviating concerns, we hold bi-yearly meetings with residents whereby we inform them about the research, remind them what the sensors tell us, assure them that we are not interested in 'watching' anyone person in particular and that all data is anonymised and only one or two key researchers have the 'keys' to identify people from participant ids. Despite this, there are still concerns 3 years on. This highlights the need for constant communication with residents and reassurance.

Prior to moving to GNH, only 2 of the residents had previously used computers. Now the residents partake in regular iPad classes run by researchers at GNH. "Once you know the basics you find out for yourself" "Oh I'm flying now", "And me using Skype and emails and everything - it's brilliant." One resident referring to his grandson "He's a genius on it. He shows me everything." However, it was agreed that training and support is essential in learning how to use new techologies such as the $\mathrm{iPad}$, to provide initial confidence.
As we further our research at $\mathrm{GNH}$, we will continue to work closely with the residents and to continually evaluate and improve the applications they use. Critically, we will examine how the feedback we are providing affects behaviour and wellbeing longitudinally.

\section{Case Studies}

This section highlights two case studies of the research that is ongoing at GNH. The studies are in the areas of sleep and emotional wellbeing. The importance of these wellness parameters for older adults is described in the following sections, along with details of how they are implemented within our closed-loop system at GNH.

\subsection{Sleep}

Sleep is a fundamental physiological process with important restorative functions. Sleep problems have been shown to be detrimental to human health. In humans, short (seven hours or less) and long (nine hours or more) durations of sleep have been shown to be significant predictors of death in prospective population studies [8]. Sleep disturbances may be indicative of poor health and functional deficits, especially in older adults [39], [41]. Total sleep time is reduced in the elderly and this is not due to a reduced need for sleep, but in a diminished ability to sleep [1]. Sleep complaints are commonly reported by over $50 \%$ of those aged 65 and older [41]. These complaints include getting less sleep, frequent awakenings, waking up too early, excessive daytime sleepiness, and napping during the day. Decreased quality of life, and higher rates of depression and anxiety are reported in patients with sleeping difficulties [2]. In direct comparisons against matched controls, aged patients with sleep difficulties have significant cognitive impairment and limited attention spans. Additionally, high incidences of balance, ambulatory and visual difficulties (after controlling for medication use) have been reported in older adults with sleep problems [4]. Furthermore, decreased total sleep time (TST), an increased sleep latency (SL), defined as the time taken to fall asleep in bed, and a poor sleep efficiency (SE), defined as the percentage of TST over total time in bed (TIB), are linked to a greater risk for mortality (even when controlling for related covariates) [19]. Additionally, the symptoms of various chronic conditions continue 


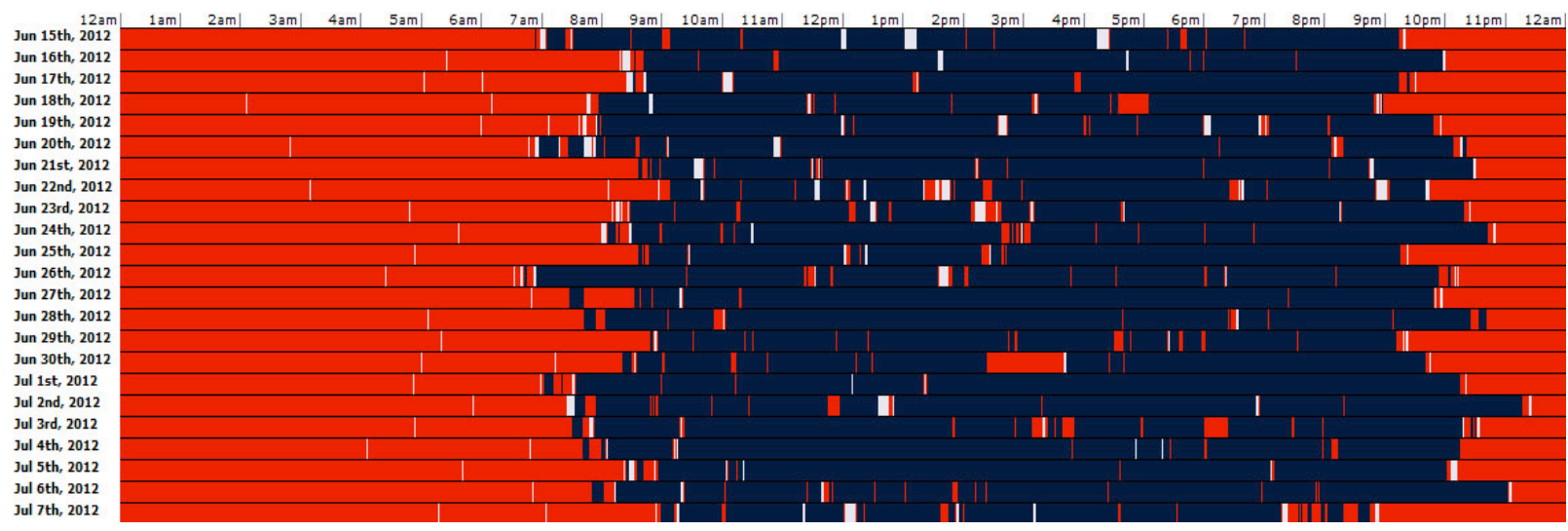

Figure 14. Good sleeper (red areas represent time in the bedroom; pale grey represents time in the en suite; and navy represents time in other areas of the house)

into the night and result in a disturbed sleep; these include movement disorders, neuromuscular diseases, depression, dementia, epilepsy, obesity and circadian rhythm disorders [23].

The gold standard sleep assessment technology is polysomnography (PSG) which records multiple physiological signals (including brain activity, muscle tone, eye movements, heart rate and respiration) during sleep. PSG is intrusive, costly, time consuming and often results in sleep which is atypical of natural behaviour. Wrist actigraphy is the current ambulatory gold standard sleep monitoring device. It consists of a two axis accelerometer which records the rest/activity patterns of the wearer and converts this to sleep/wake estimates (using slightly modified thresholding algorithms). Wrist actigraphy has been shown to estimate nocturnal sleep duration and sleep-wake patterns reliably where PSG is not a suitable alternative [35], [58]. However, a low wake detection capacity is often reported with this device as it cannot discriminate between quiescent wake and sleep [51]. Wrist actigraphy is dependent on the adherence (and conscious participation) of the wearer. Sleep diaries are also used (often concomitantly with wrist actigraphy) to estimate sleep duration in normal, institutionalised and pediatric populations. However their validity relies upon the attentiveness of the individual filling the diaries out (in cases where the diaries are filled out by the individual). A trade-off exists for these technologies between accuracy and suitability for long term deployment.

An ambient living technology approach offers a more practical solution for long term sleep monitoring and will ideally avoid a requirement for interaction with the subject. Many techniques have been developed to this end including video-based solutions [43], [50], radar based technologies [17], load-cell movement detection sensors placed under bed posts [5], [12], pressure pads placed on top of the mattress [38], under mattress sensors [6], [9], [34], [59], [60], [67] and pillow based sensors [69]. Many of these systems are particularly suited to non-contact long-term sleep monitoring as they do not require specialist expertise to install. However, some systems have usability constraints ranging from limitations in a practical deployment to a rejection of the technology by the participants (for example, due to privacy concerns, the noticeable presence of the sensor when in bed, comfort, etc.)

\subsubsection{Ambiently Assessing Sleep}

PIR based monitoring systems [13], [60] have been developed with a high accuracy being reported. A number of potential usability issues have been raised, relating to varying location of heaters between environments and the presence and types of bed sheets shielding sensor from the minute movements of the individual. However, PIR based techniques have the capacity to monitor longitudinal changes in sleeping patterns unobtrusively over extended periods of time. The layout of GNH, including the underfloor heating system, facilitates the collection of movement patterns of an individual from which daily patterns of rest and activity are extracted. Through comparing current patterns with subjectspecific data, unhealthy patterns of behaviour (in terms of daily patterns such as an increased time in bed) can be highlighted and used to guide an intervention.

To date measures have been derived for bed time, rise time, time in bed and sleep disturbances (as 
described in Section 3.4). Initial validation of these algorithms has been undertaken whereby two healthy adults with no sleep complaints and three older adults, reporting a poor sleep quality (PSQI > 5), stayed for a period of between 2 and 4 days in the Great Northen Haven test/demonstration apartment. Each participant wore an actiwatch (MotionWatch 8, CamNtech, Cambridge, UK) for the duration of their stay and completed a number of clinically validated questionnaires to establish ground truth.

Measures for bed time, rise time and time in bed, derived from the sensor data, were compared with equivalent measures derived from the actiwatch data and PSQI questionnaire results. Table 1 shows the results of the comparison, averaged per subject over multiple nights, between the quiescent (or assumed sleep) time in bed derived from the PIR sensors (TIB-S) and actual sleep time from the actiwatch (TIB-A) data, as well as the time in bed derived from the answers to questions 1 and 3 of the PSQI questionnaire (TIB-P), which relate to bed time and wake time over the past month.

These results report close alignment between TIB$A$ and TIB-S for four of the five participants, particularly the healthy adults. The mean absolute error (MAE), when comparing the sensor against the actiwatch measures for the older male participant reports an error of 0.71 hours (43 mins); ongoing work is investigating the use of subject-specific movement thresholds to improve results. A distinct difference is seen between the subjectively reported time-in-bed (TIB-P) and both the TIB-A and TIB-S. Furthermore, the older adult female (1) scored high on the PSQI compared to the male (14 vs 6), denoting a worse subjective sleep quality than the male. The results of the two healthy participants are quite different, in comparison to those of the older adults, indicating that the TIB measure may be more accurate for good sleepers.

From this initial investigation, we are beginning to see that across all subjects, TIB-S aligns more closely with to TIB-A, whereas TIB-P does not align well with either TIB-S or TIB-A. However, this doesn not negate the importance of TIB-P (or TIB-A). Both remain important indicators for clinicians [49].

Table 1. Comparison of self-reported sleep, actigraphy-defined sleep, and CASALA-defined sleep

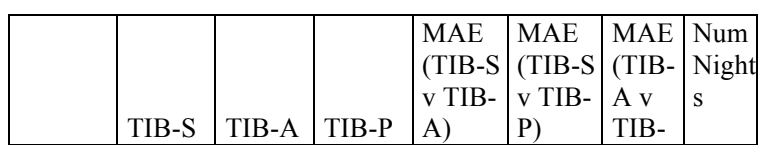

\begin{tabular}{|l|l|l|l|l|l|l|l|}
\hline & & & & & & $\mathrm{P})$ & \\
\hline $\begin{array}{l}\text { Older } \\
\text { Female } \\
1\end{array}$ & 9.68 & 9.4 & 12.75 & 0.283 & 3.06 & 3.35 & 4 \\
\hline $\begin{array}{l}\text { Older } \\
\text { Male }\end{array}$ & 5.50 & 6.21 & 9.25 & 0.71 & 3.74 & 3.03 & 4 \\
\hline $\begin{array}{l}\text { Older } \\
\text { Female } \\
2\end{array}$ & 7.65 & 8.04 & 8.5 & 0.388 & 0.84 & 0.45 & 3 \\
\hline $\begin{array}{l}\text { Healthy } \\
\text { Female }\end{array}$ & 7.68 & 7.57 & 8 & 0.10 & 0.31 & 0.42 & 4 \\
\hline $\begin{array}{l}\text { Healthy } \\
\text { Male }\end{array}$ & 9.06 & 9.06 & 8.5 & 0.00 & 0.56 & 0.56 & 2 \\
\hline
\end{tabular}

When applying this algorithm to 16 of the permanent residents of GNH, a comparison was undertaken between the good sleepers (see Table 2) and poor sleepers (see Table 3). The TIB-P results relate to questions 1 and 3 of PSQI questionnaires gathered on two separate occasions, during June (questionnaire 1) and November (questionnaire 2) 2012. Each TIB-S value was calculated using the mean TIB derived from the PIR sensors for the month prior to questionnaires being administered and so is directly comparable to the TIB-P scores. The mean absolute error indicates that the TIB-S for good sleepers is predominantly lower than the equivalent results for the poor sleepers when compared with their subjective sleep duration.

Table 2. Comparison of self-reported sleep and CASALA-defined sleep for good sleepers (PSQI $\leq 5$ )

\begin{tabular}{|l|c|c|c|c|}
\hline ID & Questionnaire & TIB-S & TIB-P & $\begin{array}{c}\text { MAE (TIB-S } \\
\text { v TIB-P) }\end{array}$ \\
\hline 2 & 1 & 8.540 & 8.5 & 0.040 \\
\hline 3 & 1 & 9.150 & 9.25 & 0.100 \\
\hline 4 & 2 & 11.065 & 11.5 & 0.435 \\
\hline 9 & 2 & 10.913 & 10.5 & 0.413 \\
\hline 9 & 1 & 9.569 & 9 & 0.569 \\
\hline 11 & 1 & 8.623 & 9.5 & 0.877 \\
\hline 25 & 2 & 8.108 & 8.5 & 0.392 \\
\hline
\end{tabular}

Table 3. Comparison of self-reported sleep and CASALA-defined sleep for poor sleepers (PSQI $>5$ )

\begin{tabular}{|l|c|c|c|c|}
\hline ID & Questionnaire & TIB-S & TIB-P & $\begin{array}{l}\text { MAE (TIB-S } \\
\text { v TIB-P) }\end{array}$ \\
\hline 2 & 1 & 8.324 & 9 & 0.676 \\
\hline 7 & 2 & 5.476 & 5 & 0.476 \\
\hline 7 & 1 & 6.146 & 6.5 & 0.354 \\
\hline 8 & 1 & 8.594 & 9 & 0.406 \\
\hline 11 & 2 & 8.130 & 10.25 & 2.120 \\
\hline 12 & 1 & 8.632 & 9 & 0.368 \\
\hline 12 & 2 & 9.710 & 10 & 0.290 \\
\hline 20 & 1 & 7.361 & 7.25 & 0.111 \\
\hline 20 & 2 & 7.016 & 8.5 & 1.484 \\
\hline 22 & 2 & 8.297 & 8 & 0.297 \\
\hline
\end{tabular}


However, a further validation is ongoing whereby residents wear an actiwatch for a period of a week, complete the daily survey each day and finally fill out a PSQI to determine the sleep quality for the validation period. This is investigating optimal methods of tuning subject-specific thresholds for the ambient system. As validation is still in progress, sleep-related feedback does not currently consider measures derived from the sensor data.

\subsubsection{Location Mapping}

Through analysing sensor firings sequentially, the movements of the residents between rooms can be monitored. Significant changes in these patterns over time may highlight abnormal behaviours and may signal an impaired health status. Higher level features can be derived from this location information. For example, a map of residents location allows for prolonged periods spent in the bedroom to be grouped. These periods may then be used to derive measures for bed time, rise time, time in bed and resultantly number, duration and destination of disturbances. Figures 14 and 15 show the resulting location maps, for two residents (one poor sleeper where PSQI $>5$, and one good sleeper $\leq 5$ ). The red areas represent the time the resident was in the bedroom, the pale grey represents time spent in the en suite, and navy represents time spent in other areas of the house. Other features of interest are number of room transitions per day, time outside the home etc.

\subsubsection{Sleep Disturbances}

Sleep disturbances have been classed according to their location, including movement within the bedroom, movement to the ensuite bathroom and movement to other areas of the house. While the location maps in Figures 14 and 15 look quite similar in terms of their duration in the bedroom at night, the poor sleeper has a lot of sleep disruption, both in terms of movement between rooms and movement within the bedroom. This movement is clearly visible when comparing the movement levels in the bedroom, between 12 am and $12 \mathrm{pm}$, of the good sleeper, Figure 16 (a) with that of the poor sleeper, Figure 16 (b). The good sleeper has disruptions to sleep to go to the bathroom however they only have a short duration of movement within the bedroom directly before and after these bathroom visits.

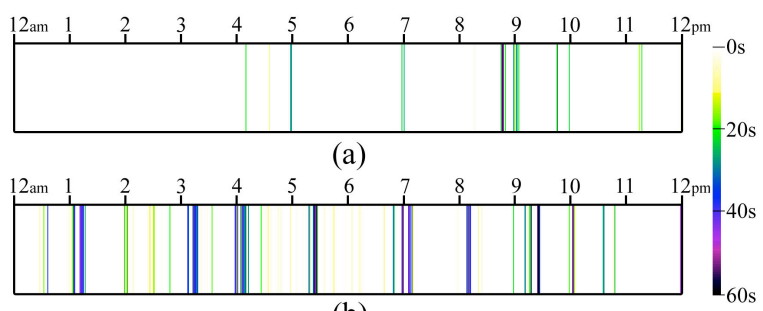

(b)

Figure 16. movement levels per minute of a good sleeper (a) and poor sleeper (b) between midnight and midday

\subsection{Emotional Wellbeing}

The term mental health, while sometimes used to denote mental or emotional wellbeing, is most often used to mean mental illness and indeed lay people have equated the term mental health with mental illness [52]. For this reason, we use the term emotional wellbeing throughout this article. There are many definitions of emotional wellbeing. The

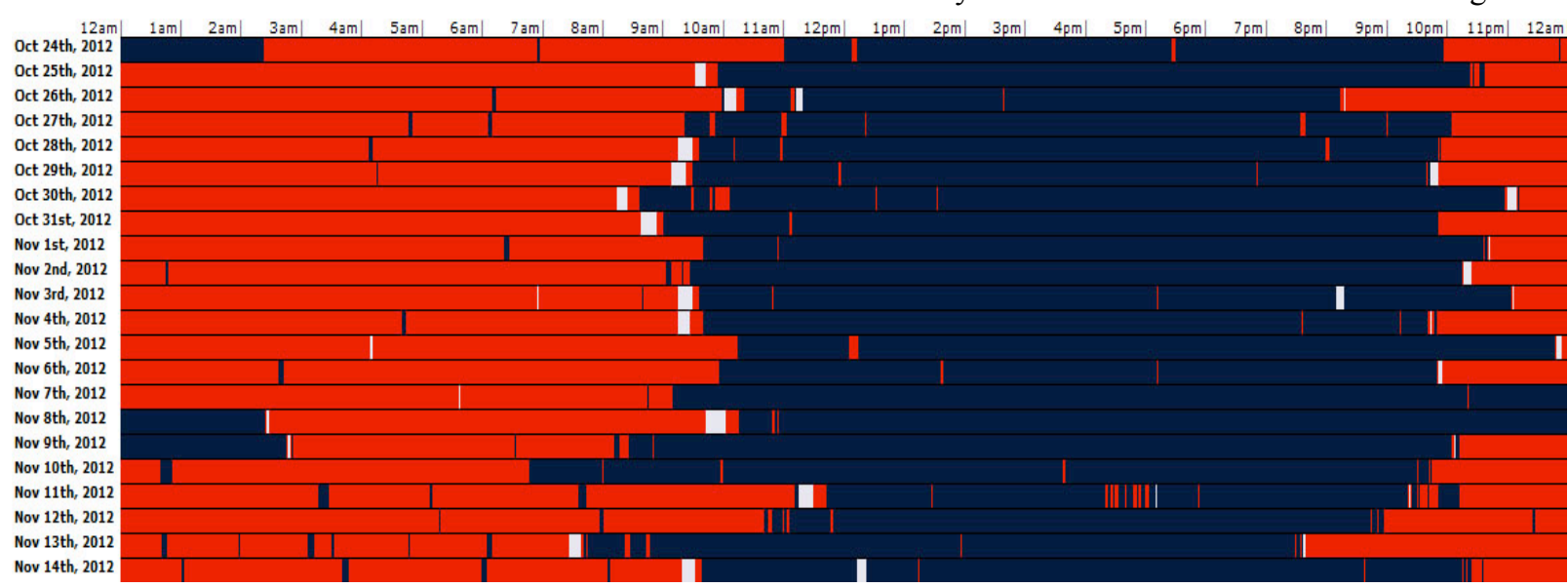

Figure 15. Poor sleeper (red areas represent time in the bedroom; pale grey represents time in the en suite; and navy represents time in other areas of the house) 
Mental Health Foundation in the UK defines positive mental or emotional health as the ability to make the most of your potential, to cope with life and to play a full part in your family, workplace, community and among friends [40]. A definition from the NHS Scotland in their national mental health strategy for 2012-2015 includes areas such as life satisfaction, optimism, self-esteem, mastery and feeling in control, having a purpose in life and a sense of belonging and support [47].

Emotional wellbeing is an important indicator of overall health in adults over 65 [21], [45]. Yet, within the field of technology design for older adults to support independence, emotional wellbeing is often overlooked, with research tending to focus on the three 'geriatric giants' of healthy ageing - physical, cognitive and social health. For some older people, age-related declines to physical, cognitive or social wellbeing can negatively impact on their emotional wellbeing, as can anxiety, loneliness and depression resulting from the notion of growing older, the loss of a spouse, a loss of sense of purpose or general worries about coping, becoming ill and/or death. However, the reverse is also true - poor emotional wellbeing can equally adversely affect one's overall health and wellbeing. Fair to poor emotional wellbeing has been shown to be significantly associated with poor appetite in older adults [21] which in turn will affect their physical wellbeing.

Furthermore, many chronic health problems faced by older adults have a high rate of co-occurring depression. Up to $25 \%$ of people with cancer suffer depression, as do up to $27 \%$ of people who have had a stroke and 1 in 3 people who have suffered a heart attack [45]. It is estimated that up to $5 \%$ of US citizens over the age of 65 living in the community have major depression compared with $13.5 \%$ of those who require home health care and $11.5 \%$ in nursing homes [25]. However, a significantly larger number suffer depression that remains largely undiagnosed and thus untreated [57]. Technology-based systems could therefore help older adults to monitor and selfmanage their emotional wellbeing by providing feedback and interventions that can in turn be used to promote positive emotional wellbeing and perhaps, more significantly, improvements to overall wellbeing.

\subsubsection{Ambiently Assessing Emotional State}

A person's emotional wellbeing state is typically assessed using clinically validated questionnaires such as the Center for Epidemiologic Studies
Depression Scale (CES-D) [53] or the Geriatric Depression Scale (GDS) [68]. The issue with such assessments in practice is that they are typically reliant on clinical professionals to administer them and thus are only delivered when a person visits their clinician. Developing mechanisms to shift aspects of these assessments, as well as treatment of emotional disorders from clinical settings to self-management in the home, and simultaneously providing continuous, in-depth, histories emotional wellbeing state changes between clinical visits is preferable.

Ambient sensing has the potential to support monitoring and prediction of changes to emotional state from within the home. We have recently begun to examine this within GNH. Figures 17 and 18 shows data for two GNH residents derived from the PIR sensors within the home to determine a person's (1) activity level within the home and (2) time outside the home. To help visualise behavioural patterns the sensor data is represented on a polar plot called a "last clock". They show data on a 24-hour clock with midnight at the top and noon at the bottom. Day 1 begins in the centre with each concentric circle outwards representing each subsequent day. Activity level is represented by the blue colour, whereby periods of blue indicate activity and periods of white indicate no activity. Larger white blocks indicate bed time or time outside the home, while larger blue blocks represent times of continuous activity. These particular images represent data collected over a 30-day period. Complete circles indicate when a resident was away from the home.

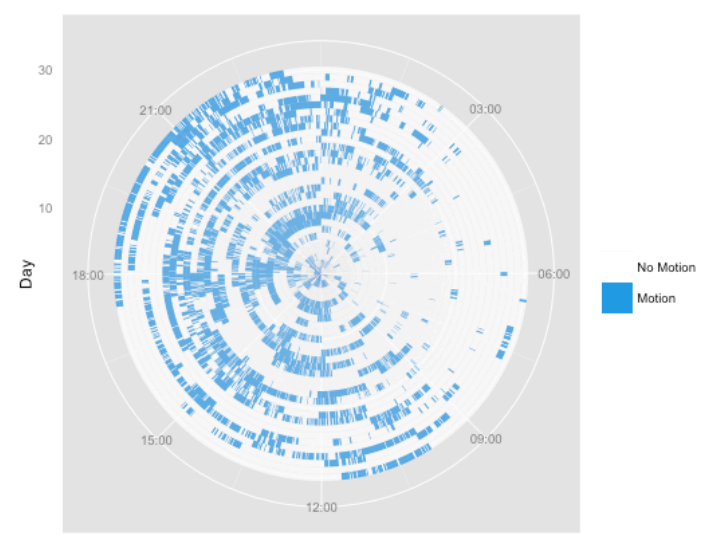

Figure 17. Clock plot for a resident with high levels of activity 
The clock plot in Figure 17 shows data for an 'active' resident who spends a significant portion of their time outside the home and is often active while inside the home. Figure 18 shows a contrast where movement levels are shown for an 'inactive' resident who spends a significant portion of their time inside the home and is not very active while in the home. Baseline data gathered from the resident in Figure 18 illustrates high levels of depression. Interviews with this resident suggest that possible reasons behind the depression include worries about declining health but primarily a lack of social contact with others.

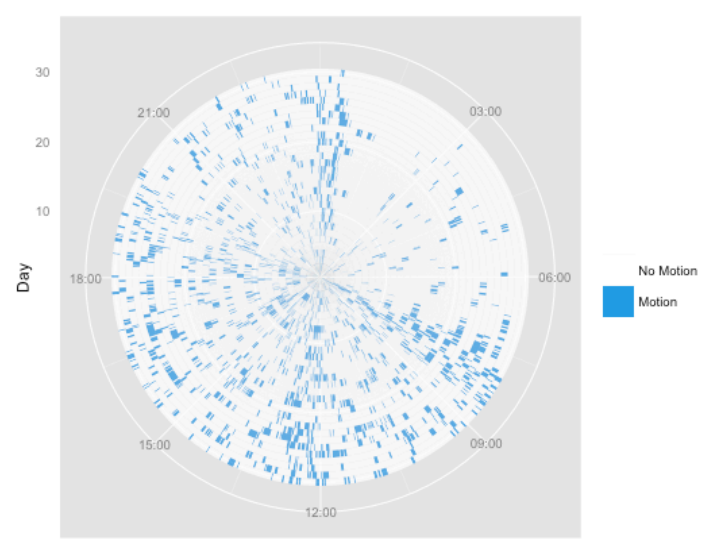

Figure 18. Clock plot for resident with low levels of activity

Thus a visual comparison of these two clock plots provides us with a picture of the behavioural differences between two people. However, ground truth data is necessary to know that these graphs depict the behaviour of someone who is emotionally unwell compared with someone who is emotionally healthy.

What we are particularly interested in is looking for changes to a person's wellbeing state over time and intervening in a timely manner, to prevent a person from moving to an 'inactive' or unhealthy state of being. Our next steps in ambiently assessing changes to emotional wellbeing involve integrating additional metrics that might be indicators of declining emotional wellbeing, including decreased or erratic movement levels and increased time in bed. Correlating these metrics with self-reported emotional wellbeing from the iPad wellness application and our quarterly clinical questionnaires will allow us to determine the most effective measure of emotional state.

\subsection{Self-report Assessment}

The self-report assessment for emotional wellbeing and sleep is made through the YourWellness iPad application, described earlier. As discussed in Section 3.4.7, the self-report assessment acts as ground truth for the sensing data in addition to supporting the assessment of the performance and predictive power of the system through comparative analysis. For emotional wellbeing, we aim to examine correlations of ambient sensing with selfreported mood and satisfaction with social interactions. Similarly, we will examine correlations between self-reported sleep and ambient objective measures. This will provide a finer granularity than comparisons with our quarterly questionnaires. The self-report assessment can be remotely updated with new questions, individualised for each resident to help us understand their patterns of behaviour and 'why' certain changes might be occuring.

\subsection{Feedback}

Currently, feedback provided through the iPad wellness application is derived from the self-reported data as described above. As discussed in section 3.5.1, the feedback provided consists of an overview of ones's wellbeing, trends over time and a series of educational messages aimed at increasing the older person's awareness of their wellbeing and supporting them in maintaining a healthy state of wellbeing. For example, some messages to support positive emotional wellbeing include 'Physical health is connected to emotional health. Try taking a 30 minute walk each day'; 'Don't skip breakfast...or any other meal. Studies show that people who eat breakfast report being in a better mood and have more energy throughout the day'. Sleep messages include 'Nap early in the day, or not at all. Late-day naps decrease sleep drive. If you must nap, it's better to keep it short and before 5 p.m.'; 'Avoid caffeine, alcohol, nicotine and other chemicals that interfere with sleep for 4-6 hours before bedtime'. Initial testing of this application suggests that such messages are useful to people and not necessarily already known. A thorough evaluation of the effectiveness of the feedback will be conducted in the coming months. 


\section{Discussion/Lessons Learned}

This section highlights some of the lessons we have learned during our work at GNH which we feel will benefit other researchers in the area of smart environments to support AAL. While some are more practical in nature, and may seem obvious to the reader, we believe they will help researchers in this space avoid trial and error scenarios.

Plan for sensor failure and ongoing maintenance

When designing systems that will monitor residents in their own homes it is important to plan for failure. Sensor failures, computer hardware failures, power outages, network outages and databases reaching their maximum size all need to be detected and planned for. The hardware failures need an engineer to come on site to deal with the problem so an ongoing service contract is useful.

\section{Cater for externally-driven system downtime}

In a wired sensor network power outages lead to data loss but are so infrequent that they are not a major issue. Network outages are dealt with by having a local database on site which caches the sensor readings and from which data is replicated.

\section{Understand database limitations}

Database size limitation can be overcome by replicating the data and deleting data that has been replicated from the local box. In order to process the data efficiently it is necessary to keep the size of database tables low enough to ensure that they can fit in memory. This is achieved by partitioning the data in both daily tables and monthly tables.

\section{Recognise that access to residents' home will be infrequent, at best}

Once the system is deployed there is very limited physical access to the homes so it is useful to have a test apartment with the same layout as the resident's homes in which to test sensors and algorithms.

\section{Create automated reports highlighting sensor failure}

It is necessary to constantly monitor the quality of the data coming in to the system as well as to understand the data. This allows us to detect when hardware or systems fail and to act to fix them. The complexity of validating individual sensors depends on the type of data that they report. Periodic sensors are easy to validate as they should report a constant number of records in any time period. Sensors which report periodically and on change can be validated by making sure that they report a minimum number of records in any time period. On change only sensors can be validated by looking at a sliding window of the past 30 days and seeing that the sensor is firing within its normal range.

\section{Systematically review sensor installation}

Mapping out the visibility of PIRs also allows us to understand the data that they are reporting. Originally the PIR sensors were located in the corner of the living room, facing the main entry point to the apartment and in the corner of the bedroom. While these picked up on most movement within the rooms they missed out on transitions between rooms. Consequently movement from the living room through the hallway into the bedroom was often missed. Consequently, the location of these sensors was changed on 18th April 2011. Another visibility issue was highlighted during PIR testing of newly installed sensors. The orientation of the sensor in question provided very poor visibility. Once identified it was altered and retested resulting in full visibility being achieved. This emphasises the need for validating the visibility and effectiveness of sensors.

In developing the location mapping algorithm the PIR's overshoot time was found to affect certain scenarios. When checking for movement between rooms, for example, an issue arose whereby if an individual moved from one room (room A) to another (room B) and back to room A, prior to the PIR in room $A$ reseting to a 0 value, the location would report the individual's location as room B instead of room A, as the PIR in room B was triggered after the PIR in room A. The PIR's overshoot time also affected detection of movement to adjacent rooms which are undetectable to PIRs. An individual is deemed to be in an adjacent room between the time the movement ceased in the PIR visible room and the time it recommences. An allowance is required when a sensor used to detect presence in an adjacent room is located within a PIR's line of sight.

\section{Understanding resident behaviour is essential}

Once we have validated the raw data we extract features on which to build models. These range from the simple of finding total energy usage from firings every twelve seconds to the much more complex of trying to assess the quality of a resident's sleep or whether the resident is depressed. We do not need 
any input from the resident to measure their energy usage.

In order to understand the more complex features such as sleep or depression it is necessary to get the resident's help in labelling the data. The wellness app described above, allows us to ask each individual resident targeted questions about their sleep and wellbeing, which will allow us to find correlations between features extracted from the sensor data and sleep quality and mood.

Estimating sleep pattern over extended durations using ambient systems must be validated, and must cater for subject specific changes over time

Estimation of sleep and sleeping patterns has been shown using the rest/activity patterns of the residents, extracted using the PIR motion detectors, as a proxy. To date, comparisons have been made against wrist actigraphy (as PSG, even ambulatory PSG, is not a practical alternative) using five subjects. Promising results were found for four participants, while sleep was underestimated (by 43 minutes) by our sleep detection algorithm for the older male subject. This is as a result of a non-subject-specific threshold being used to the join, or exclude, a duration spent in the bedroom, before or after a sleep disturbance, in calculating the sleep window, as discussed in Section 0 . Further validation of this system is required, and is underway. For this work, actigaphy data is currently being collected from the remaining residents in Great Northern Haven. The primary focus of this work lies in creating automated algorithms which define subject specific movement thresholds, and in refining the sleep detection algorithm. This will allow the implementation of similar systems in other homes with different individuals, and will also cater for long-term changes in health status and physical activity over extended periods of time.

\section{A tradeoff between specificity and suitability of long-term placement is required}

A limitation of using movement-based sleep detecting devices, including wrist actigraphy, is the ability to correctly classify periods of quiescent wake in bed [51]. Higher accuracy would be achieved using PSG, however this is inappropriate for certain populations, and over extended periods of time. As such, a trade-off exists between accuracy and the practicality of real-world deployment. Smart home technologies can be used to implement a realisable solution, particularly solutions such as that described in this paper.
Subjective and objective sleep assessments may capture different, yet important, health information

The subjective sleep duration, reported using the PSQI, conflicted with the objective estimates from the actigraphy watch and our sleep detection system. This may be attributed more generally to the use of subjective questionnaires. For example, the PSQIdefined time in bed (calculated as the difference between bed time and wake time over the past month) for each of the older adults $(12.75,9.25$ and 8.5 hours) also conflicted largely with the PSQIreported sleep duration answers (3, 7 and 3.66 hours). In such cases, sleep state misperception or personality type may play a large role.

The power of the objective non-contact sleep detection method reported herein does not solely lie in reporting the approximate sleep time on a per night basis, but also in reporting longitudinal deviations in sleeping patterns. Ongoing work is currently relating such variations to measures of overall health status. Additionally, ongoing work is relating the fragmentation of rest/activity profiles, derived from the motion detectors, against measures of emotional wellbeing, loneliness, depression, anxiety, cognition, and quality of life. In particular, longitudinal deviations in the fragmentation of rest/activity will be investigated.

\section{Involving residents at all stages keeps them engaged in the research}

The residents of GNH are at the centre of everything that we do. We hold regular meetings with them to update them on the research we are doing and to understand their needs and requirements. We use a mixed-methods approach in working with the residents, from indivudual interviews to focus groups and co-design workshops involving some or all of the residents. This is essential, from learning how they feel about selfmanagement to understanding how we can support them in interpreting and benefitting from feedback. To support technology usage and understanding, we run weekly iPad classes with the residents, ensuring they gain full benefit from the iPads and can access and use various applications in addition to our own. We have found that the residents are more engaged when they feel they have an active and important role to play in the research, rather than just being 'subjects' or 'participants'.

Help the residents to feel comfortable about ambient monitoring. 
The residents understand that there is a trade-off between privacy and what they describe as an increased feeling of security and safety that the ambient monitoring provides. We have found that one way to ensure residents feel comfortable is to remind, or reassure them (during our regular meetings) about the data that is being collected. For example, we remind them that the sensors are not cameras by visually showing them how we (the researchers) see the data. We remind them that data is anonymised and that only certain people have access to this data. Assuring the residents that they are in control of their data and that it ultimately is returned to them as feedback, is beneficial.

Feedback is essential in supporting selfmanagment

We have learned that feedback to the person themselves is important for keeping the residents engaged with the research. It reduces the feeling of being monitored and provides a sense of ownership over the data for the resident. Thus, if the person is physically and cognitively capable, then they should receive feedback in the first place. They should also decide who, if anyone, else should have access to their data, e.g. family members, clinicians. Feedback is thus essential in closing the loop in such AAL systems and in supporting self-management within the home.

\section{Conclusion and Future Work}

While research on sensor systems and detecting patterns of behaviour dominates the AAL research space, the challenge of predicting changes and prompting positive preventative intervention measures, aiding the avoidance of severe physical or mental harm, has not adequately been addressed. This paper has presented details of an integrated approach to address this issue, combining ambient sensor monitoring, self-reported data and behaviour recognition with timely educational and interventional feedback. Our research is presented within the context of 16 smart homes, where older people are living their daily lives while being supported in self-managing their wellbeing.

We are beginning to see evidence that behavioural pattern recognition techniques applied to passive data acquisition from a constellation of low-cost sensors installed in homes can provide prediction of wellbeing state. We anticipate that through machine learning techniques, pattern-recognition algorithm prediction of wellbeing state changes can converge within zones of tolerance to feedback from selfreported and assessment instruments, increasing confidence in ambient prediction. Early evidence indicates that the delivery of informational, educational and interventional feedback to the person themselves can support them in managing their wellbeing and encourages behaviour change where necessary to promote positive wellbeing. Future work will evaluate the effectiveness of such feedback longitudinally. In our ongoing and future work, we expect that validated interventions can transfer from laboratory, clinical and healthcare settings to home environments and that their integration will support positive behavioural state changes. Our research is also forming the basis of a new commercial venture, CASAcloud. CASAcloud is a communications platform empowering direct stakeholders involved in ageing-in-place to shift from traditional emergency response models to proactive models of early interventions, prevention and wellness, powered by unique context aware inferences encapsulated in an applications framework.

\section{Acknowledgements}

The authors would like to acknowledge funders of this research including Atlantic Philantropies and Enterprise Ireland, the latter of whom funded CASALA under the Applied Research Enhancement programme. We also acknowledge Dundalk Town Council and the Health Service Executive of Ireland who are partners on the Great Northern Haven facility.

\section{References}

[1] S. Ancoli-Israel. Sleep problems in older adults: Putting myths to bed. Geriatrics, 52(1):20-29, 1997.

[2] S. I. Barbar, P. L. Enright, P. Boyle, D. Foley, D. S. Sharp, H. Petrovitch, and S. F. Quan. Sleep disturbances and their correlates in elderly japanese american men residing in hawaii. J Gerontol A Biol Sci Med Sci, 55(7):M406-M411, Jul 2000.

[3] A. Bhattacharya and S.K. Das. LeZi-Update: an information-theoretic approach to track mobile users in PCS networks. In Proceedings of the 5th Annual ACM/IEEE International Conference on Mobile Computing and Networking (MobiCom'99), (1999).

[4] G. S. Brassington, A. C. King, and D. L. Bliwise. Sleep problems as a risk factor for falls in a sample of community- 
dwelling adults aged 64-99 years. J Am Geriatr Soc, 48(10):1234-1240, Oct 2000.

[5] M. Brink, C. H. Mller, and C. Schierz. Contact-free measurement of heart rate, respiration rate, and body movements during sleep. Behav. Res. Methods, 38(3):511521, Aug 2006.

[6] C. Brueser, K. Stadlthanner, S. de Waele, and S. Leonhardt. Adaptive beat-to-beat heart rate estimation in ballistocardiograms. IEEE Trans. Inf. Technol. Biomed., 15(5):778-786, Sep 2011

[7] D.J. Buysse, C.F. Reynolds, T.H. Monk, S.R. Berman and D.J. Kupfer, The Pittsburgh Sleep Quality Index: a new instrument for psychiatric practice and research. In Psychiatry Res. 28(2) (1989), pp. 193-213.

[8] F. P. Cappuccio, L. D'Elia, P. Strazzullo, and Michelle A. Miller. Sleep duration and all-cause mortality: a systematic review and meta-analysis of prospective studies. Sleep, 33(5):585-592, May 2010.

[9] B. W. Carlson, V. J. Neelon, and H. Hsiao. Evaluation of a non-invasive respiratory monitoring system for sleeping subjects. Physiol Meas, 20(1):53-63, Feb 1999.

[10] CDC National Vital Statistics Reports, Vol. 61, No. 6 Deaths: Preliminary data for 2011, 2012.

[11] C. Chen, B. Das, and D. Cook. A data mining framework for activity recognition in smart environments. Proceedings of the International Conference on Intelligent Environments, (2010).

[12] J. H. Choi and D. K. Kim. A remote compact sensor for the real-time monitoring of human heartbeat and respiration rate. Biomedical Circuits and Systems, IEEE Transactions on, 3(3):181-188, June 2009.

[13] M. Choi, B. O. Kim, B. S. Hwang, R. H. Sohn, and K. S. Park, Unobtrusive body movement monitoring during sleep using infrared motion detector and zigbee protocol. In Proc. 3rd IEEE/EMBS International Summer School on Medical Devices and Biosensors, (2006), pp. 32-36.

[14] D. Cook, M. Schmitter-Edgecombe, A. Crandall, C. Sanders, and B. Thomas. Collecting and disseminating smart home sensor data in the CASAS project. Proceedings of the CHI Workshop on Developing Shared Home Behavior Datasets to Advance HCI and Ubiquitous Computing Research, (2009).

[15] CSO Ireland report "Ageing in Ireland", 2007.

[16] S.K. Das, D.J. Cook, A. Bhattacharya, E.O. Heierman, T.Y. Lin. The role of prediction algorithm in the MavHome smart home architecture. In IEEE Wireless Commun., 9, (6), (2002), pp. 77-84.

[17] P. de Chazal, E. O'Hare, N. Fox, and C. Heneghan. Assessment of sleep/wake patterns using a non-contact biomotion sensor. Engineering in Medicine and Biology Society, 2008. EMBS 2008. 30th Annual International Conference of the IEEE,pages 514-517, Aug. 2008.

[18] J. de Jong Gierveld, and F.H. Kamphuis, The development of a Rasch-type loneliness-scale. In Applied Psychological Measurement, 9, (1985), pp. 289-299.

[19] M. A. Dew, C. C. Hoch, D. J. Buysse, T. H. Monk, A. E. Begley, P. R. Houck, M. Hall, D. J. Kupfer, and C. F. Reynolds, 3rd. Healthy older adults' sleep predicts all-cause mortality at 4 to 19 years of follow-up. Psychosom Med, 65(1):63-73, 2003.

[20] J. Doyle, B. O'Mullane, S. McGee and B. Knapp, B, YourWellness: Designing an application to support positive emotional wellbeing in older adults. In BCS HCI 2012 (2012).

[21] J.H. Engel, F. Siewerdt, R. Jackson et al. Hardiness, depression and emotional wellbeing and their association with appetite in older adults. Journal of American Geriatrics Society, 59, 3, (2011), pp. 482-487.

[22] English Longitudinal Study of Ageing, Wave 3, 2005/06.

[23] S Happe. Excessive daytime sleepiness and sleep disturbances in patients with neurological diseases. Drugs, 63(24):2725-2737, 2003.

[24] S. Helal, W. Mann, H. El-Zabadani, J. King, Y. Kaddoura, and E. Jansen, The gator tech smart house: A programmable pervasive space.Computer, 38(3), (2005), pp. 50-60.

[25] C.F. Hybels and D.G. Blazer, Epidemiology of late-life mental disorders. Clinical Geriatric Medicine, 19, 4, (2003), pp. 48-51.

[26] S.S. Intille, Designing a home of the future. Pervasive Computing, IEEE, 1(2), (2002), pp. 76-82.

[27] Irish Department of Health, Health Statistics 2005. Section B - Life Expectancy and Vital Statistics.

[28] G. Jain, D. Cook, and V Jakkula, Monitoring health by detecting drifts and outliers for a smart environment inhabitant. In Proceedings of the international conference on smart homes and health telematics, (2006), pp. 114-121.

[29] H. Jimison and R. Bajcsy, Integrated Communications and Inference Systems for Continuous Coordinated Care of Older Adults in the Home. NSF Collaborative Research Grant. Accessed on: 12th Dec. 2012 http://www.orcatech.org/research/studies/integratedcommunications-and-inference-systems-for-continuouscoordinated-care-of-older-adults-in-the-home.

[30] O. Kafali, S. Bromuri, M. Sindlar, T. van der Weide et al. COMMODITY12: A smart e-health environment for diabetes management. In Journal of Ambient Intelligence and Smart Environments, 5, (5), (2013), pp. 479-502.

[31] J.A. Kaye, S.A. Maxwell, N. Mattek, T. Hayes, Dodge H, Pavel M, Jimison H, Wild K, Boise L, \& Zitzelberger T. Intelligent Systems for Assessing Aging Changes: HomeBased, Unobtrusive and Continuous Assessment of Aging. Journal of Gerontology: Psychological Sciences. 2011; 66B(suppl 1): i180-i190.

[32] C.D. Kidd, R. Orr, G.D. Abowd, C.G. Atkeson, I.A. Essa, B. MacIntyre, E.D. Mynatt, T. Starner, and W. Newstetter, The Aware Home: A Living Laboratory for Ubiquitous Computing Research. In Proceedings of the Second International Workshop on Cooperative Buildings, Integrating Information, Organization, and Architecture (CoBuild '99), (1999), Springer-Verlag, pp. 191-198.

[33] J.A. Kientz, S.N. Patel, B. Jones, E. Price, E.D. Mynatt, and G.D. Abowd, The Georgia Tech aware home. In CHI'08 Extended Abstracts on Human Factors in Computing Systems (CHI EA '08). (2008), ACM, pp. 3675-3680.

[34] J.M. Kortelainen, M.O. Mendez, A.M. Bianchi, M. Matteucci, and S. Cerutti. Sleep staging based on signals acquired through bed sensor. IEEE Trans. Inf. Technol. Biomed., 14(3):776-785, May 2010.

[35] C. A. Kushida, A. Chang, C. Gadkary, C. Guilleminault, O. Carrillo, and W. C. Dement. Comparison of actigraphic, polysomnographic, and subjective assessment of sleep parameters in sleep-disordered patients. Sleep Med, 2(5):389-396, Sep 2001.

[36] M.P. Lawton and E.M. Brody, Assessment of older people: self-maintaining and instrumental activities of daily living. In Gerontologist 9(3), (1969), pp. 179-86.

[37] B. Logan, J. Healey, M. Philipose, E. M. Tapia, and S. Intille, A long-term evaluation of sensing modalities for activity recognition. In Proceedings of the 9th international conference on Ubiquitous computing (UbiComp '07), (2007), Springer-Verlag, Berlin, Heidelberg, 483-500. 
[38] D. C. Mack, J. T. Patrie, P. M. Suratt, R. A. Felder, and M. A. Alwan. Development and preliminary validation of heart rate and breathing rate detection using a passive, ballistocardiography-based sleep monitoring system. IEEE Trans. Inf. Technol. Biomed., 13(1):111-120, Jan 2009.

[39] K. Manabe, T. Matsui, M. Yamaya, T. Sato-Nakagawa, N. Okamura, H. Arai, and H. Sasaki. Sleep patterns and mortality among elderly patients in a geriatric hospital. Gerontology, 46(6):318-322, 2000.

[40] Mental Health UK. http://www.mentalhealth.org.uk/

[41] L.E. Miles and W. C. Dement. Sleep and aging. Sleep, 3(3):119-220, 1980.

[42] M.C. Mozer, The neural network house: an environment that's adapts to its inhabitants. In Proceedings of the AAAI Spring Symposium on Intelligent Environments, Technical Report SS-98-02, (1998), pp. 110-114.

[43] K Nakajima, Y Matsumoto, and T Tamura. Development of real-time image sequence analysis for evaluating posture change and respiratory rate of a subject in bed. Physiological Measurement, 22:N21-N28, 2001.

[44] Z.S. Nasreddine, N.A. Phillips, V. Bédirian, S. Charbonneau, V. Whitehead, I. Collin, J.L. Cummings, H. Chertkow, The Montreal Cognitive Assessment, MoCA: A Brief Screening Tool For Mild Cognitive Impairment. In Journal of the American Geriatrics Society, 53 (4), (2005), pp. 698-699.

[45] National Institute of Mental Health: Depression Publications. http://www.nimh.nih.gov/index.shtml. (retrieved August 2012).

[46] NHS Nice Guidelines. http://www.nice.org.uk

[47] NHS Scotland. http://www.healthscotland.com/mentalhealth-background.aspx

[48] A. O' Brien, K. McDaid, et al. (2012). Visualisation of Movement of Older Adults within their Homes based on PIR Sensor Data. In PERVASENSE, Workshop at Pervasive Health '12, San Diego, United States, IEEE.

[49] M.M. Ohayon and J. Zulley (2001). Correlates of global sleep dissatisfaction in the German population. In Sleep, 24 (7), pp. 780-787.

[50] S. Okada, Y. Ohno, Goyahan, K. Kato-Nishimura, I. Mohri, and M. Taniike. Examination of non-restrictive and noninvasive sleep evaluation technique for children using di_erence images. Conf Proc IEEE Eng Med Biol Soc, 2008:3483-3487, 2008.

[51] J. Paquet, A. Kawinska, and J. Carrier. Wake detection capacity of actigraphy during sleep. Sleep, 30(10):13621369, 2007.

[52] S. Pavis, H. Masters and S.C. Burley, Lay concepts of positive mental health and how it can be maintained. University of Edinburgh, (1996).

[53] L.S. Radloff, The CES-D scale. A self-report depression scale for research in the general population. In Applied Psychological Measurement, 1, (3), (1977), pp. 385-401.

[54] P. Rashidi, D.J. Cook, L.B. Holder and M. SchmitterEdgecombe, Discovering Activities to Recognize and Track in a Smart Environment,. In Knowledge and Data Engineering, IEEE Transactions on , vol.23, no.4, (2011), pp.527-539.

[55] S. Robben, M. Boot, M. Kanis and B. Krose. Identifying and visualizing relevant deviations in longitudinal sensor patterns for care professionals. In Pervasive Health ' 13 workshop on Lifelogging for Pervasive Health.

[56] S. Robben, G. Englebienne, M. Pol and B. Krose. How is Grandma doing? Predicting functional health status from binary ambient sensor data. In AAAI Technical Report FS12-01 Artificial Intelligence for Gerontechnology, pp. 2631.

[57] J.A. Sable, L.B. Dunn, and S. Zisook, S, Late-life depression. How to identify its symptoms and provide effective treatment. In Geriatrics, 57, (2000).

[58] A. Sadeh, K.M. Sharkey, and M.A. Carskadon. Activitybased sleep/wake identification: An empirical test of methodological issues. Sleep, 17:201-207, 1994.

[59] J. H. Shin, Y. J. Chee, D. Jeong, and K. S. Park. Nonconstrained sleep monitoring system and algorithms using air-mattress with balancing tube method. IEEE Trans. Inf. Technol. Biomed, 14(1) (2010), pp. 147-156.

[60] J. W. Shin, Y. R. Yoon, and J. C. Principe. Evaluation of body movement during sleep by thermopile using wavelet and neuro-fuzzy. In Proc. 25th Annual International Conference of the IEEE Engineering in Medicine and Biology Society, (2003), pp. 2406-2407.

[61] Si4Life Living Lab. http://www.si4life.it. Accessed December 2012.

[62] M. Skubic, R. Guevara and M. Rantz, M, Testing classifiers for embedded health assessment. Impact Analysis of Solutions for Chronic Disease Prevention and Management, (2012), pp. 198-205.

[63] E-E. Steen, T. Frenken, M. Eichelberg, M. Frenken and A. Hein. Modelling individual healthy behaviour using home automation sensor data: Results from a field trial. In Journal of Ambient Intelligence and Smart Environments, 5, (5), (2013), pp. 503-523.

[64] E.M. Tapia, S.S. Intille and K. Larson, Activity Recognition in the Home Setting Using Simple and Ubiquitous Sensors. In Proceedings of PERVASIVE 2004, vol. LNCS 3001, (2004), pp. 158-175.

[65] US Census Bureau National Population Projections, 2008.

[66] J.E. Ware Jr, and C.D. Sherbourne, The MOS 36-item shortform health survey (SF-36). I. Conceptual framework and item selection. Med Care., 30(6):473-83, June, 1992.

[67] T. Watanabe and K. Watanabe. Noncontact method for sleep stage estimation. Biomedical Engineering, IEEE Transactions on, 51(10):1735-1748, 2004.

[68] J.A. Yesavage, T.L. Brink, T.L. Rose, O. Lum et al., Development and validation of a geriatric depression screening scale: A preliminary report. In Journal of Psychiatric Research, 17, (1980), pp. 37-39.

[69] X. Zhu, W. Chen, T. Nemoto, Y. Kanemitsu, K.-I Kitamura, K.-I. Yamakoshi, and D. Wei. Real-time monitoring of respiration rhythm and pulse rate during sleep. Biomedical Engineering, IEEE Transactions on, 53(12):2553-2563, Dec. 2006.

[70] A.S. Zigmond and R.P. Snaith. The hospital anxiety and depression scale. In Acta Psychiatr Scand. 67, (6), (1983), pp. 361-70. 